\title{
Maximum stress of stiff elastic plate in uniform flow and due to jet impact
}

\author{
A.A. Korobkin ${ }^{1}$, T.I. Khabakhpasheva ${ }^{2}$, S. Malenica ${ }^{3}$ \\ ${ }^{1}$ School of Mathematics, University of East Anglia, Norwich, UK, \\ e-mail: a.korobkin@uea.ac.uk \\ ${ }^{2}$ Lavrentyev Institute of Hydrodynamics, Novosobirsk, Russia \\ e-mail: tana@hydro.nsc.ru \\ ${ }^{3}$ Bureau Veritas, Marine \& Offshore Division - Research Department, France, \\ sime.malenica@bureauveritas.com
}

The liquid jet impact onto a clamped elastic plate is investigated. The two-dimensional jet of constant thickness and with flat vertical front is initially advancing towards the elastic plate along a flat, rigid and horizontal plane at a constant uniform speed. The elastic plate of variable thickness is mounted perpendicular to the rigid plane. The maximum stress during the early impact stage is estimated for a given retardation time and a given relaxation time of the plate material. The stresses during the initial impact stage are compared with the static stresses in the plate placed in an equivalent uniform flow. It is shown that the static stresses are always smaller than the bending stresses during the early stage of impact for a given speed and thickness of the jet. This implies that if the stresses in the plate are smaller than the yield stress of the plate material with no plastic deformations in the plate occurring during the unsteady impact stage, then the plate behaves elastically after the impact and plastic deformations are not achieved. Approaching the plastic deformations is treated here as a damage to the plate. The maximum stress increases with the increase of the jet thickness. A critical value of the jet velocity, below which the plate is not damaged by the jet impact, is obtained for given characteristics of the plate.

\section{Introduction}

The two-dimensional problem of fluid impact onto a clamped elastic plate is considered. The flow region before impact is a semi-strip bounded from below by a rigid plane and above by the flat free surface. The front free surface of the flow region is vertical, see figures 1a and 1b. We distinguish two cases with the plate being longer than the jet thickness (figure 1a) and the plate shorter than the jet thickness (figure 1b). The fluid is advancing initially towards the elastic plate at a constant uniform speed. The elastic plate is clamped to the rigid bottom at its lower end. The upper end of the plate is free of stresses. The plate deflection is described by the Euler beam equation. We are concerned with the bending stresses in the plate caused by impact. In particular, we shall determine the maximum speed of the jet impact, at which the plate still behaves elastically with the bending stresses in the plate being below the value of the yield stress of the plate material. The problem is coupled: the plate deflection and the hydrodynamic loads acting on the plate are determined simultaneously. We consider elastic plates of high rigidity such that the plate deflection caused by plate interaction with the fluid is relatively small and the lowest period of the plate free vibration in the air is also small. The maximum stresses are achieved during an early stage of short duration. During the initial impact stage, see figures $1 \mathrm{c}$ and $1 \mathrm{~d}$, the problem is linearized and solved by the normal mode method at leading order. Later on both the plate deflection and the flow around the plate approach their steady states with the flow separating from the upper end of the plate and forming a cavity behind the plate, see figures $1 \mathrm{e}$ and $1 \mathrm{f}$. The stresses in the plate at this later stage are determined by decoupled approach and used as reference stresses in the present analysis. 

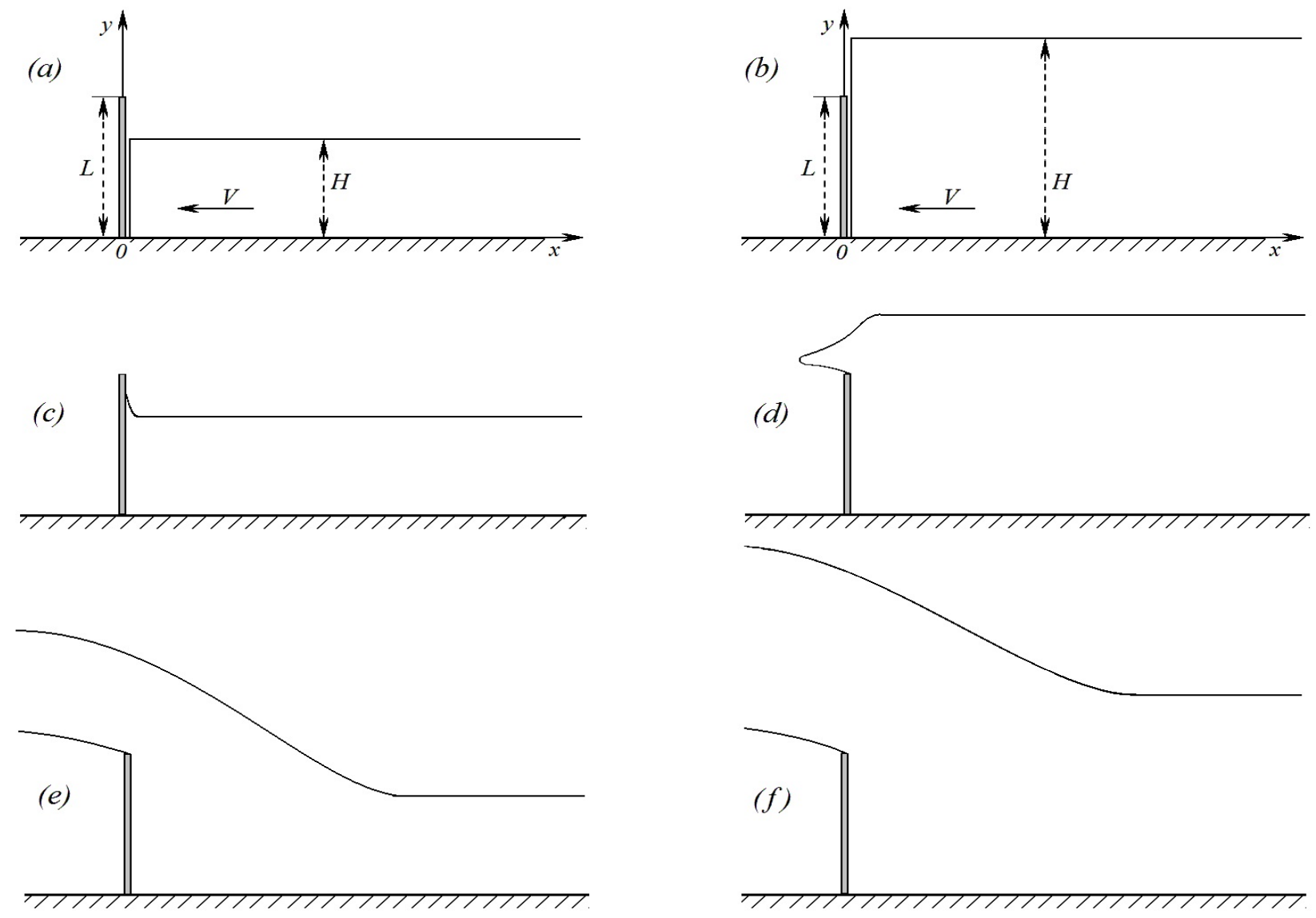

Fig. 1 Jet impact onto elastic clamped plate: (a, b) positions of the plate and the liquid region before impact, (c, d) sketches of the flows during the initial stage of impact, (e, f) sketches of the steady flows during the later stage.

This study is motivated by the problem of violent sloshing in liquefied natural gas (LNG) tanks of NO96 type. NO96 membrane system is a cryogenic liner which includes two metallic membranes and two insulation layers ${ }^{1}$. The membranes are made of $500 \mathrm{~mm}$ wide and $0.7 \mathrm{~mm}$ thick strips of a nickel-steel alloy. The strips of the primary membrane, which is in contact with the LNG cargo, are joined by welding their raised edges with a tongue between them (figure $2 \mathrm{a}$ ). The tongue

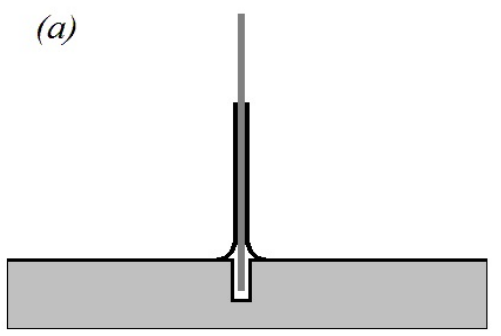

(b)

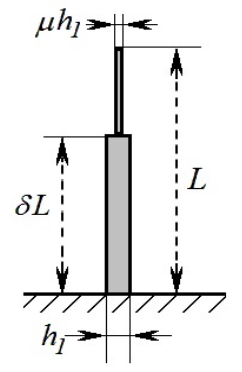

Fig. 2 The tongue of NO96 system of length $L$ and with the raised edges of length $\delta L$ :

(a) sketch of the tongue and the raised edges, (b) dimensions of the NO96 system. 
is made of the same alloy but it is $0.5 \mathrm{~mm}$ thick. It goes above the raised edges of the primary membrane and also below the primary level. The tongues allow sliding the membrane on the top of the insulation layer avoiding shear stresses in the system. Also the transverse loads caused by LNG sloshing are reduced through deflections of the tongue and the raised edges. We shall determine critical velocities of the LNG near a tongue, which may lead to plastic deformations of either raised edges of the primary membrane or the tongue itself, in uniform flow and during jet impact onto the tongue. Jet-type flows can be generated by sloshing wave impact onto the tank wall near the tongue. The jet speed can be much greater than a typical speed of the main flow. This problem can be also related to sloshing wave impact on corrugations of Mark-III containment system ${ }^{2}$. However, the Mark-III corrugations cannot be approximated by thin plates. Shallow-water sloshing in a 2D tank with a typical elastic panel of Mark-III containment system was studied both experimentally and numerically by Lugni et al. ${ }^{3}$ The walls of the tank were rigid with the elastic panel mounted near the equilibrium water level. The study was concerned with the flip-through type of sloshing impact which is characterized by hydrodynamic loads of short duration and very high magnitude.

Experimental, theoretical and numerical studies of sloshing-induced impact loads on tank walls of LNG vessels involve many complex physical phenomena such as phase transition, fluid compressibility and aeration. Smoothed Particle Hydrodynamics (SPH) simulations of liquid impact on complex structures like Mark-III and NO96 containment systems were performed by Oger et. $\mathrm{al}^{4}$. Several test cases were used to validate the developed SPH structural model, including the case of deformable beam wedge impacting the free surface at very high speed. The full-scale sloshing experiments using focused waves impacting on a fully instrumented LNG carrier NO96 membrane containment panel are described by Brossest et. $\mathrm{al}^{5}$. These experiments led to new insights into sloshing impact and the influence of hydro-elasticity. The paper by Lafeber et. al ${ }^{6}$ shows that different regimes of interaction between breaking waves and corrugated wall induce loads that can be presented as combination of direct impact, building jet along the wall and compression/expansion of entrapped gas elementary loading processes. The configuration of the present study corresponds to the direct impact elementary process ${ }^{6}$.

At larger scale of order of few meters, the problem of this study corresponds to that of tsunami bore impact on coastal structures and interaction of dry-bed surges and broken waves with buildings ${ }^{7,8}$. Some tsunamis may break offshore and arrive at the coast as a tsunami bore ${ }^{9}$ with almost uniform both depth and speed of the flow. Surge waves are also resulting from dam breaks. The profile of the bore front could be rather steep, see the free-surface elevation in figure 3 of Wei et al. ${ }^{8}$, and can be approximated by a vertical front. The flows in surge waves and broken waves are very turbulent ${ }^{10}$ with significant amount of air entrained before the impact onto a structure. The fluid near the wave front is aerated ${ }^{11,12}$. However, it was shown ${ }^{13}$ that the maximum stresses in an elastic wall impacted by a breaking wave are weakly dependent on the level of the fluid aeration.

The present model of jet impact onto vertical elastic plate is highly simplified in terms of the impact conditions. In reality, the jet front is not parallel to the plate at impact instant (figure 3a), compressibility (figure 3b) and aeration of the fluid in the impact region (figure 3c), as well as the presence of the air in between the plate and the approaching jet front (figure $3 \mathrm{~d}$ ), matter. These effects make the hydrodynamic loading on the plate to be gradual in time and can be described, in terms of the maximum bending stress in the plate, by using a concept of retardation time, which characterizes the duration of the early transient stage. The idea behind the concept of the retardation time is that the complex problem of fluid impact onto an elastic plate in practical situations can be split into two: (1) the problem of jet impact onto a vertical elastic plate with the jet front being vertical and the retardation time given, (2) estimation of the retardation time for a particular situation. The concept of the retardation time is also related to the condition of the 

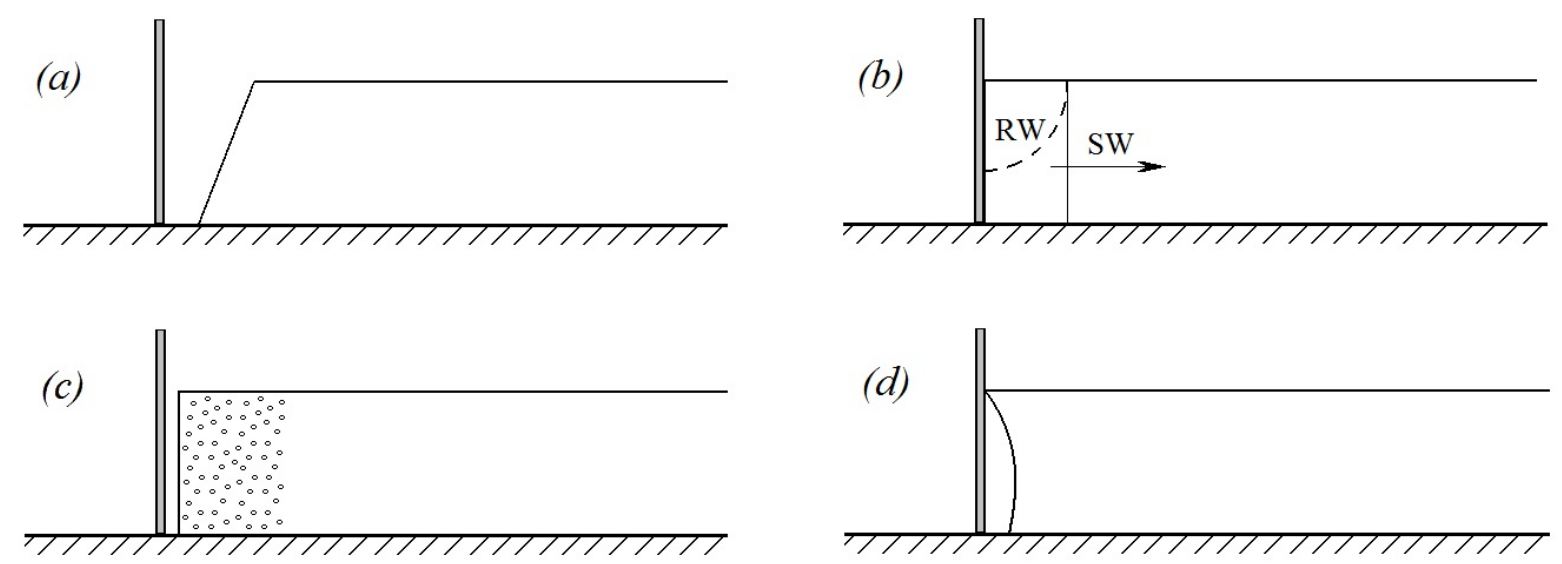

Fig. 3 The jet impact onto a vertical elastic plate with (a) the jet front not parallel to the plate, (b) compressible liquid in the jet, (c) aerated front of the jet, (d) presence of the air in between the plate and the approaching jet front.

plate failure by the jet impact. In the present model, it is required that the bending stress in the plate should stay above the yield stress value for a certain time before plastic deformations start. Note that we assume here that the elastic model of the plate can still be used for stresses near and slightly higher than the yield stress value.

The problem of a clamped elastic plate in a jet flow is formulated in section II. The list of nomenclature used in the paper is placed just after References. The elastic plate is of non-constant thickness. Only the plates with piecewise constant thickness are simulated but the theoretical model and the algorithm of its analysis are valid for more complicated structures with almost flat surface. The stationary stresses in the plate with the flow separation at the plate edge are estimated in section III within the decoupled approach. These stresses are used as the reference stresses for the jet impact problem in section IV within the coupled theory of hydroelasticity. Calculations are performed for the parameters of the tongue shown in figure 2. The obtained numerical results are presented in section V. It is shown that the steady-state stresses are much smaller than the maximum stress achieved during the impact stage. The conclusions are drawn in section VI. We conclude, in particular, that if an elastic plate has survived the impact stage without plastic deformations, then it will not be damaged in the subsequent steady flow.

\section{Formulation of the problem}

The two-dimensional interaction between a fluid jet of thickness $H$ and an elastic plate of length $L$ is considered in the Cartesian coordinate system $x, y$ with the origin at the clamped edge of the plate, see figure $1 \mathrm{a}, \mathrm{b}$. The fluid moves towards the plate at a uniform speed $V$. The fluid occupies the semi-infinite region, $x>0,0<y<H$, at the time of impact, $t=0$. The fluid is assumed incompressible and inviscid. Gravity and surface tension effects are neglected. The presence of air between the plate and the approaching vertical front of the jet is not included in the present analysis. The elastic plate of variable thickness $h(y)$, where $0<y<L$, is clamped to the flat bottom, $y=0$. Another end of the plate is free of stresses and shear forces. The plate is vertical and perpendicular to the direction of the flow before impact. We assume that the plate deflection from its initial position is small even at the beginning of the plastic deformations in the plate. 
Pressure-impulse theory and fully coupled approach are used to estimate the bending stresses in the plate during the early stage of impact, duration of which is of order of the main period of the free vibration of the equivalent elastic beam. Decoupled approach is used to evaluate the static stresses in the plate at large times.

The jet thickness $H$ can be smaller or greater than the plate length $L$. The jet flow can be caused by liquid impact onto a rigid wall, by breaking wave impact onto a vertical wall, or it can model a long wave propagating towards a vertical structure along a dry bed. In practical problems, the jet is of variable thickness and the jet (wave) front is not parallel to the wall at the time of impact. However, the present analysis still can be used if the jet thickness varies slowly with the distance from the wall and the jet speed is relatively large. The assumptions of the vertical wave front and incompressible liquid overestimate contributions of the higher modes of the plate vibration caused by the jet impact. To account for realistic conditions of the jet/wave impact, a retardation time $T_{r}$ is introduced below. The retardation time accounts for the fact that the hydrodynamic loads are not applied instantly to the plate due to some physical effects (see figure 3), which are not included in the present simplified model. The retardation time $T_{r}$ may also account for the fact that the plastic deformations of the plate material cannot start instantly, when the local stress achieves the yield stress value.

We shall evaluate the bending stresses in the plate caused by the jet impact and to compare them with the yield stress $\sigma_{Y}$ of the plate material. If the maximum of the induced stresses exceeds the yield stress during a time interval $\left(t-T_{r} / 2, t+T_{r} / 2\right)$, then the plate is said be damaged by impact at time instant $t$. Here $T_{r}$ is the retardation time of the plate material. In this case plastic deformations in the plate occur and the plate cannot return to its initial shape after the hydrodynamics loads are released. This condition of the plate damage is used in this study in the integral sense: the plate is damaged if the elastic stress averaged over time intervals of duration $T_{r}$ exceed the yield stress $\sigma_{Y}$ of the plate material.

Both the short-term and long-term interactions of the plate with the jet flow are studied. In the long-term analysis, the jet flow and the plate deflection are stationary, the hydrodynamic loads are much smaller than the loads during the initial impact stage but they last longer. In the short-term analysis valid for the early stage, duration of which is of the order of the period of the first mode of the free plate vibration, hydrodynamic loads are impulsive and the plate is more likely to be damaged if the jet speed is large enough. It will be shown in the present study that, if the plate has not been damaged during the impact stage, it will not be damaged in the steady jet flow.

The plate deflection, $w(y, t)$, is governed by the linear Euler beam equation

$$
\rho_{p} h(y) \frac{\partial^{2} w}{\partial t^{2}}+\frac{\partial Q}{\partial y}=p(-w(y, t), y, t), \quad \frac{\partial M}{\partial y}=Q(y, t), \quad E I(y) \frac{\partial^{2} w}{\partial y^{2}}=M(y, t) \quad(0<y<L),
$$

where $\rho_{p}$ is the density of the plate material, $Q(y, t)$ is the shear force, $M(y, t)$ is the bending moment, $E$ is the Young modulus of the plate material, $I(y)=h^{3}(y) / 12$ is the moment of the inertia of the plate sections, the plate deflection, $w(y, t)$, is positive in the direction of the flow. The plate is clamped at the lower edge and free of stresses at the upper end:

$$
w(0, t)=0, \quad w_{y}(0, t)=0, \quad Q(L, t)=0, \quad M(L, t)=0 .
$$

The hydrodynamic pressure, $p(x, y, t)$, is given by the Bernoulli equation

$$
p=-\rho\left(\frac{\partial \varphi}{\partial t}+\frac{1}{2}|\vec{u}|^{2}-\frac{1}{2} V^{2}\right), \quad \vec{u}(x, y, t)=\nabla \varphi
$$


in the flow region $\Omega(t)$, where $\varphi(x, y, t)$ is the velocity potential and $\vec{u}(x, y, t)$ is the velocity field of the flow. The velocity potential satisfies the Laplace equation, $\nabla^{2} \varphi=0$, in the flow region, the kinematic boundary condition and the dynamic boundary condition, $p=0$, on the free surface of the fluid region, the body boundary condition, $\varphi_{x}+w_{y} \varphi_{y}+w_{t}=0$, on the wet surface of the plate, $x=-w(y, t)+h(y) / 2$, the condition on the bottom, $\varphi_{y}=0$, where $y=0$ and $x>0$, the condition at infinity, $\varphi \sim-V x$ as $x \rightarrow+\infty$, and the initial condition $\varphi=-V x$ in the initial flow region $\Omega\left(0^{-}\right)$before impact, $t=0^{-}$. The formulated problem is coupled: the plate deflection depends on the hydrodynamic pressure through the beam equation (1) and the pressure depends on the plate deflection through the boundary condition on the plate surface. The stresses on the surface of the plate are given by

$$
\sigma(y, t)=\frac{1}{2} h(y) M(y, t) / I(y)=6 M(y, t) h^{-2}(y), \quad \sigma(y, 0)=0,
$$

where tensile stresses are positive. The condition that the plate is not damaged during an initial time interval $\left(0, T_{f}\right)$ is formulated as

$$
\max _{0<t<T_{f}} \max _{0 \leq y<L}\left|\frac{1}{T_{r}} \int_{t-T_{r} / 2}^{t+T_{r} / 2} \sigma(y, \tau) \mathrm{d} \tau\right|<\sigma_{Y} .
$$

The condition (5) is a simplified version of the failure criterion by Petrov and Utkin ${ }^{14}$. They argued that any known criteria of quasi-static fracture transferred directly to dynamic problems "would be physically incorrect"14. They wrote "It should be mentioned that within the limits of the force fracture mechanics, a relatively high instantaneous value of the acting force should lead to failure. However, the dynamic failure is accompanied by the change in the extent of motion of the particles adjacent to the fracture area and a force pulse is required for failure. For example, in interpreting failure separation of two "atoms" 15 , it is essential to take into account the inertia. To separate the two elements it is not sufficient to apply the single force; this force must act for a relatively long period of time." In the criterion of dynamic failure introduced in ${ }^{14}$, stresses are averaged over a time $T_{r}$, which is "the time of transfer of the interaction from one structural element to another," and over a small structural element. In the condition (5), we do not distinguish structural elements and we do not average the bending stress $\sigma(y, t)$ over such elements as it is done in ${ }^{14}$.

To model the tongue and raised edges of NO96 membrane system, we consider the plate of piecewise constant thickness, $h(y)=h_{1}$, where $0<y<\delta L$, and $h(y)=\mu h_{1}$, where $\delta L<y<L$, see figure 2. Here $0<\delta<1$ and $0<\mu<1$. The plate of constant thickness is obtained with either $\delta=1$ or $\mu=1$. For NO96 membrane system, $\mu=5 / 19$ and $\delta=4 / 7$.

\section{Decoupled problem of plate deflection in uniform flow}

The stresses in the elastic plate during the early impact stage are expected to be high due to impulsive hydrodynamic loading of large magnitude. The hydrodynamic loads after the impact stage are smaller but they last longer. We do not consider here periodic vibrations of the plate in a steady jet. It is possible that the clamped plate has survived the impact loads without plastic deformations, but it is deformed plastically later on, when the loads are stationary. In order to investigate if it is possible or not, we consider the steady problem of elastic clamped plate placed in steady uniform flow. This problem can be considered as the jet/plate interaction problem for infinite thickness of the jet. A reason for such an approximation comes from the analysis of hydrodynamic 
force acting on a rigid plate in steady jet flow. It is shown by Birkhoff, Plesset and Simmons ${ }^{16,17}$ that the total force acting on the plate is weakly dependent on the jet thickness $H$ for $H>L$. We also assume that the deflection of the elastic plate in a steady uniform flow is small and the hydrodynamic pressures along the plate can be approximated by their values calculated for an equivalent rigid plate. In the dimensional variables, the static pressure distribution along the plate, $x=0,0<y<L$, is given in parametric form by the formulae ${ }^{16}$ :

$$
p(0, y)=\frac{1}{2} \rho V^{2}\left(1-\tan ^{2}(\theta / 2)\right), \quad y=\frac{2 L}{4+\pi}(2 \sin \theta+\sin \theta \cos \theta+\theta),
$$

where the parameter $\theta$ varies from zero at the bottom, $y=0$, to $\pi / 2$ at the plate edge. Equations (6) provide

$$
p(0, y) \mathrm{d} y=\frac{4 \rho V^{2} L}{4+\pi} \cos ^{2} \theta \mathrm{d} \theta .
$$

Integrating the static beam equation, $\mathrm{d} Q / \mathrm{d} y=p(0, y)$, see the first equation in (1), in $y$ using (7) and the edge conditions (2), we find the shear force distribution along the plate:

$$
Q(y)=\frac{4 \rho V^{2} L}{4+\pi}\left(\frac{1}{4} \sin (2 \theta)+\frac{1}{2} \theta-\frac{\pi}{4}\right),
$$

where the vertical coordinate $y(\theta)$ is given by (6). Note that $p(0, y) \geq 0$ and, therefore, $Q(y)$ is a monotonically increasing function with $Q(L)=0$ and $Q(0)=-\pi \rho V^{2} L /(4+\pi)$.

The second equation in (1) in static case, $\mathrm{d} M / \mathrm{d} y=Q(y)$, provides the distribution of the bending moment along the plate. Here $Q(y) \leq 0$ and, therefore, $M(y)$ is a monotonically decreasing function with $M(L)=0$. Integrating the equation for the bending moment and using (8) and (2), we obtain

$$
\begin{gathered}
M(y)=\rho V^{2} L^{2} f(\theta), \\
f(\theta)=\frac{4}{(4+\pi)^{2}}\left\{\frac{1}{2}\left(\theta-\frac{\pi}{2}\right)\left(\sin (2 \theta)+4 \sin \theta+\theta-\frac{\pi}{2}\right)-\frac{1}{2} \cos ^{4} \theta-\frac{2}{3} \cos ^{3} \theta+\frac{1}{2} \cos ^{2} \theta+2 \cos \theta\right\}
\end{gathered}
$$

and $f(0)=4\left(\pi^{2} / 8+4 / 3\right) /(4+\pi)^{2}, f^{\prime}(\theta) \leq 0$. The non-dimensional bending moment scaled with $\rho V^{2} L^{2}$ and the shear force scaled with $\rho V^{2} L$ are shown in figure 4 as functions of the nondimensional vertical coordinate $y / L$, along the plate

The stress distribution along the elastic plate with piecewise constant thickness, $\sigma(y)$, is given by equation (4), where $\sigma(y)=\rho V^{2}\left(L / h_{1}\right)^{2} \tilde{\sigma}(y / L)$. Here $\tilde{\sigma}(y / L)$ is the non-dimensional stress on

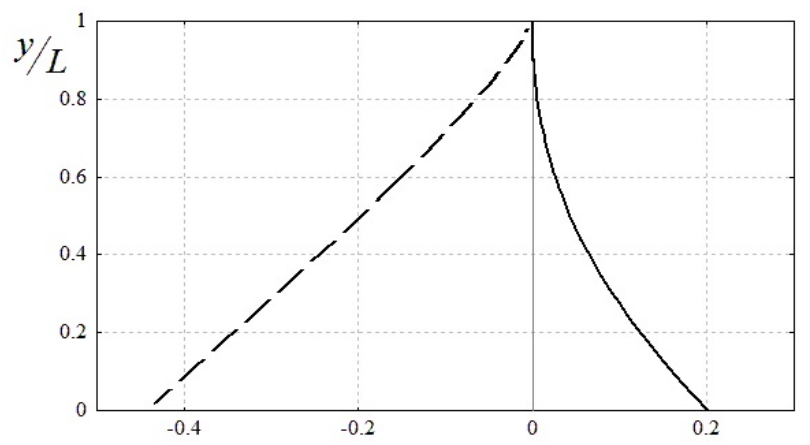

Fig. 4 Non-dimensional bending moment $M(y) /\left(\rho V^{2} L^{2}\right)$ (solid line) and shear force $Q(y) /\left(\rho V^{2} L\right)$ (dashed line) along the elastic plate. The vertical axis is for the non-dimensional coordinate $y / L$ along the plate. 

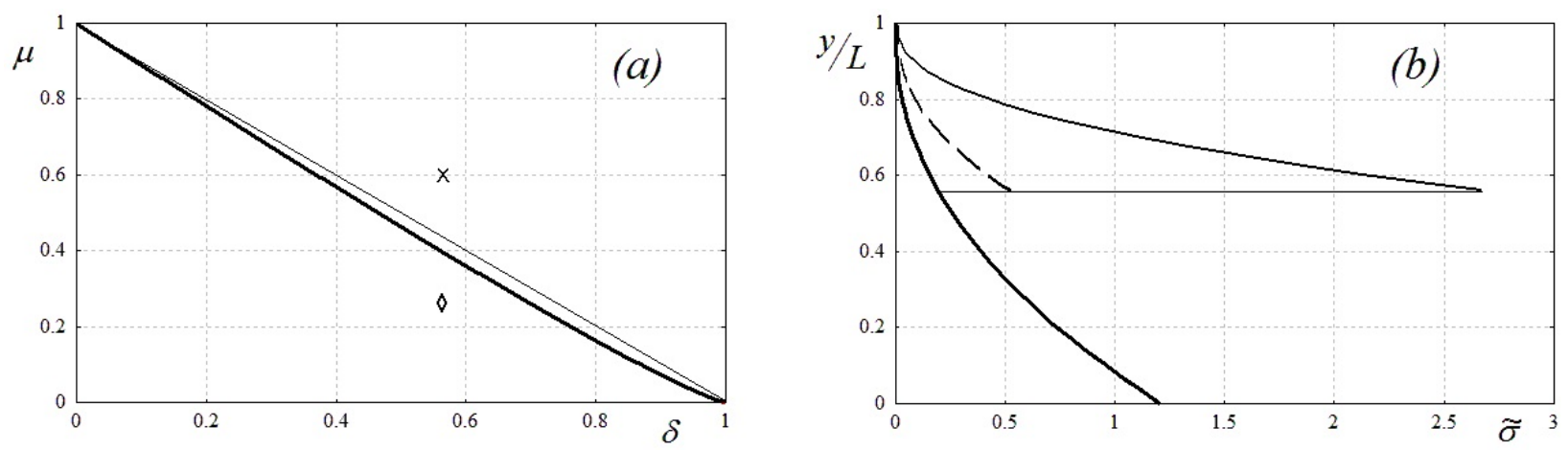

Fig. 5 (a) The function $\mu=\mu_{e}(\delta)$ (thick line) and $\mu=1-\delta$ (thin line). (b) The non-dimensional bending stress on the surface of the plate, $\tilde{\sigma}(y / L)$, as a function of the non-dimensional vertical coordinate $y / L$ for uniform plate, $\mu=1$, (thick line), tongue of NO96 system with $\mu=5 / 19$ and $\delta=4 / 7$ (thin line, diamond marker in (a)), and a tongue with $\mu=0.6$ and $\delta=4 / 7$ (dashed line, cross marker in (a)).

the surface of the plate, $\tilde{\sigma}(y / L)=6 f(\theta)$, where $0<y / L<\delta$, and $\tilde{\sigma}(y / L)=6 f(\theta) / \mu^{2}$, where $\delta<y / L<1$. Note that $\tilde{\sigma}(y / L)$ is discontinuous at $y=L \delta$. The function $f(\theta)$ is monotonically decreasing. Therefore, the maximum static stress $\tilde{\sigma}_{s, \max }$ is equal to either $\tilde{\sigma}(0)$ or $\tilde{\sigma}(\delta+0)$ depending on values of $\delta$ and $\mu$. The curve $\mu=\mu_{e}(\delta)$, on which $\tilde{\sigma}(0)=\tilde{\sigma}(\delta+0)$, is determined by the equation $\mu^{2}=f\left(\theta_{\delta}\right) / f(0)$, where $y=L \delta$ at $\theta=\theta_{\delta}$ in (6). This curve is shown in figure 5a. It is seen that $\mu_{e}(\delta) \approx 1-\delta$. Then $\tilde{\sigma}_{s, \max }=\tilde{\sigma}(\delta+0)$ for $0<\mu<\mu_{e}(\delta)$ and $\tilde{\sigma}_{s, \max }=\tilde{\sigma}(0)$ for $\mu>\mu_{e}(\delta)$. The values $\delta=4 / 7$ and $\mu=5 / 19$ corresponding to the tongue of NO96 membrane system, are shown in figure $5 \mathrm{a}$ of the $(\mu, \delta)$ - plane by diamond and the values $\delta=4 / 7$ and $\mu=0.6$ corresponding to a thicker tongue are shown by cross. The stress distributions for these tongues and for the plate of constant thickness, $\mu=1$, are shown in figure $5 \mathrm{~b}$. It is seen that the reduction of the plate thickness in $\delta<y / L<1$ increases the stresses in this interval.

The maximum static stress $\sigma_{s \text {, max }}$ is independent of the plate rigidity and is proportional to the flow speed squared. The maximum bending stress $\sigma_{s, \max }$ exceeds the yield stress of the tongue material, $\sigma_{Y}$, for the speed of the flow, $V$, such than

$$
V>\frac{h_{1} \mu}{L}\left(\frac{\sigma_{Y}}{6 \rho f\left(\theta_{\delta}\right)}\right)^{\frac{1}{2}}
$$

where $0<\mu<\mu_{e}(\delta)$, and

$$
V>\frac{h_{1}}{L}\left(\frac{\sigma_{Y}}{6 \rho f(0)}\right)^{\frac{1}{2}}
$$

where $\mu>\mu_{e}(\delta)$. Inequality (10) should be used for the tongue of the NO96 membrane system. This inequality provides that the maximum stress in the NO96 tongue exceeds the yield stress, $\sigma_{Y}=430 \mathrm{MPa}$, of the tongue material and the tongue is deformed plastically when the speed of the uniform flow of water, $\rho=1000 \mathrm{~kg} \mathrm{~m}^{-3}$, exceeds $22 \mathrm{~m} / \mathrm{s}$. For the flow of the liquified natural gas with density $\rho=468.1 \mathrm{~kg} \mathrm{~m}^{-3}$, the flow speed starting from which the tongue is deformed plastically is equal to $32 \mathrm{~m} / \mathrm{s}$. The LNG flow speed starting from which the tongue is deformed plastically at the clamped end, $y=0$, is equal to $47 \mathrm{~m} / \mathrm{s}$.

The obtained critical values of the uniform flow speed are high and difficult to be achieved in practical conditions of sloshing. The corresponding critical speed of an impulsive flow, starting from which a tongue is deformed plastically, is much smaller then the static value. The critical speed of jet impact on a tongue is estimated below. 


\section{Coupled problem of jet impact onto clamped elastic plate}

The initial stage of jet impact onto an elastic plate of variable thickness, see figure 1a and $1 \mathrm{~b}$, is considered in non-dimensional variables. We use the same notations for the non-dimensional variables but with tilde. The plate length $L$ is taken as the length scale, the product $V L$ as the scale of the velocity potential, $\rho L V / T$ as the scale of the hydrodynamic pressure during the impact stage, where $\rho$ is the fluid density, and $V T$ as the scale of the plate deflection. Here $T=2 \sqrt{3} L^{2} /\left(h_{1} c_{p}\right)$ is the time scale, $c_{p}=\left(E / \rho_{p}\right)^{\frac{1}{2}}$ is the so-called bar velocity, $E$ is the Young modulus and $\rho_{p}$ is the density of the plate material. The time-scale $T$ is proportional to the period of the first mode of the free vibration in air of the plate of constant thickness $h_{1}$. For the NO96 tongue with $E=140 \times 10^{9}$ $\mathrm{Pa}$ and $\rho_{p}=7800 \mathrm{~kg} \mathrm{~m}^{-3}$, we find $c_{p}=4236.6 \mathrm{~m} / \mathrm{s}$ and $T=5.27 \times 10^{-4} \mathrm{~s}$.

There are five non-dimensional parameters in the jet impact problem. The parameter $\varepsilon=V T / L$ is the ratio of the fluid displacement, $V T$, during the initial stage and the length scale $L$. This parameter is assumed small, $\varepsilon \ll 1$, in the present analysis. This implies that the analysis is valid for impact speeds much smaller than $c_{p} h_{1} / L$. This gives $V \ll 230 \mathrm{~m} / \mathrm{s}$ for the NO96 tongue. The small parameter $\varepsilon$ can be considered as a parameter of linearization. The boundary conditions of the hydrodynamic part of the coupled jet impact problem can be linearized at leading order as $\varepsilon \rightarrow 0$ and imposed on the positions of the liquid boundaries just before the impact, $t=0^{-}$. In particular, the non-linear Bernoulli equation in the non-dimensional variables, $\tilde{p}(\tilde{x}, \tilde{y}, \tilde{t})=-\tilde{\varphi}_{\tilde{t}}+\varepsilon\left(\tilde{\varphi}_{\tilde{x}}-\frac{1}{2}|\tilde{\nabla} \tilde{\varphi}|^{2}\right)$, where $\varphi=-V x+V L \tilde{\varphi}(\tilde{x}, \tilde{y}, \tilde{t})$ is the velocity potential of the flow, can be linearized for small $\varepsilon$ giving $\tilde{p}(\tilde{x}, \tilde{y}, \tilde{t})=-\tilde{\varphi}_{\tilde{t}}$ at leading order. The non-linear terms in the equations of motions and boundary conditions can be approximately neglected during the early stage of impact, if the impact velocity $V$ is not very high.

The plate response to the jet impact strongly depends on the jet thickness. This effects is described by the non-dimensional parameter $\gamma=H / L$, where $0<\gamma<\infty$. If $\gamma<1$, then the hydrodynamic pressure is applied only along the wetted part of the elastic plate, $0<y<\gamma$. Third non-dimensional parameter $\alpha=\rho L /\left(\rho_{p} h_{1}\right)$ indicates importance of the added mass of the plate, which is proportional to the product $\rho L$, compared to the structural mass $\rho_{p} h_{1}$ per unit length of the plate. The fourth parameter, $\tau$, is the non-dimensional retardation time of the plate material, $\tau=T_{r} / T$. The fifth parameter, $\tau_{D}$, is a non-dimensional stress relaxation time which describes structural damping of the elastic plate within the Kelvin-Voigt internal damping model ${ }^{18}$. Within this model the second term in the Euler beam equation (1) is changed to $\left(1+\tau_{D} T \partial / \partial t\right)(\partial Q / \partial y)$. This model of structural damping is "not adequate to completely represent the behaviour of real material" 18 . More complicated models of damping combining the simple Kelvin-Voigt and Maxwell models with several parameters can describe accurately responses of actual materials ${ }^{18}$. In this paper we use the simplest model of structural damping having in mind that the numerical algorithm developed below is flexible and can potentially accommodate any linear damping model. In the present analysis, $\alpha=O(1)$ and both $\tau$ and $\tau_{D}$ are small. For the NO96 tongue and LNG, we have $\alpha=1.1055$.

Initial stage of the impact with $\tilde{t}=O(1)$ is considered. The bending stresses in the plate (4) increase during the first quarter of the first period of the plate vibration in contact with the fluid, $T_{1 / 4}$, which is estimated as $T_{1 / 4} \sim \frac{2}{\pi} \alpha^{\frac{1}{2}} T$, and oscillate thereafter with decreasing in time amplitude owing to the structural damping of the plate. For the NO96 tongue, we have $T_{1 / 4} \sim 3.5 \times 10^{-4} \mathrm{~s}$.

During this early stage the plate deflection and the flow caused by impact are described in the non-dimensional variables by equations ${ }^{19,20,21}$ (tilde is dropped below)

$$
h(y) \frac{\partial^{2} w}{\partial t^{2}}+\frac{\partial^{2}}{\partial y^{2}}\left(h^{3}(y) \frac{\partial^{2} w}{\partial y^{2}}\right)=\alpha p(0, y, t) \quad(0<y<1)
$$




$$
\begin{gathered}
w(0, t)=\frac{\partial w}{\partial y}(0, t)=0, \quad \frac{\partial^{2} w}{\partial y^{2}}(1, t)=\frac{\partial^{3} w}{\partial y^{3}}(1, t)=0, \quad w(y, 0)=0 \\
\nabla^{2} \varphi=0, \quad p=-\varphi_{t} \quad(x>0,0<y<\gamma), \\
\varphi_{y}(x, 0, t)=0, \quad \varphi(x, \gamma, t)=0 \quad(x>0), \\
\varphi_{x}(0, y, t)=\chi(t)-w_{t}(y, t) \quad(0<y<1), \\
\varphi \rightarrow 0 \quad(x \rightarrow \infty),
\end{gathered}
$$

where $\chi(t)$ is the Heaviside step function, $\chi(t)=0$, where $t<0$, and $\chi(t)=1$, where $t>0$, and the plate thickness $h(y)$ in the non-dimensional variables is equal to 1 , where $0<y<\delta$, and equal to $\mu$, where $\delta<y<1$. If $\gamma>1$, then there is also the condition on the vertical part of the free surface above the plate,

$$
\varphi(0, y, t)=0 \quad(1<y<\gamma)
$$

Within the Kelvin-Voigt damping model ${ }^{18}$, equation (12) reads

$$
h(y) \frac{\partial^{2} w}{\partial t^{2}}+\left(1+\tau_{D} \frac{\partial}{\partial t}\right) \frac{\partial^{2}}{\partial y^{2}}\left(h^{3}(y) \frac{\partial^{2} w}{\partial y^{2}}\right)=\alpha p(0, y, t) \quad(0<y<1)
$$

The distribution of the non-dimensional stress along the right-hand surface of the plate is given by (4) and (1) as

$$
\sigma(y, t)=h(y) \frac{\partial^{2} w}{\partial y^{2}}
$$

with the scale $\sqrt{3} \rho_{p} c_{p} V$. The structural problem without damping, (12) and (13), and the hydrodynamic problem (14) - (18) for $\gamma>1$ are depicted in Figure 6.

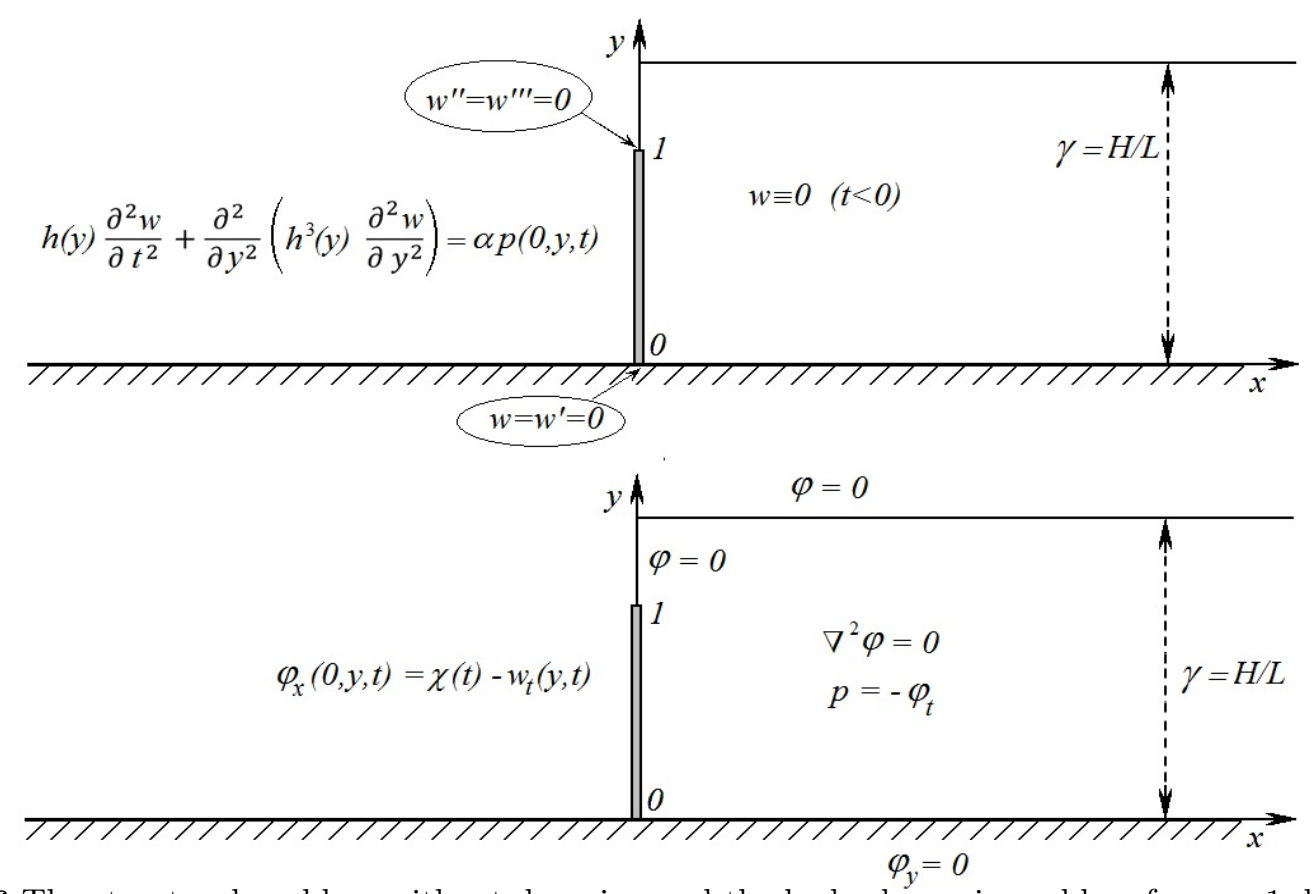

Fig. 6 The structural problem without damping and the hydrodynamic problem for $\gamma>1$ depicted together with the notations of the problems. 

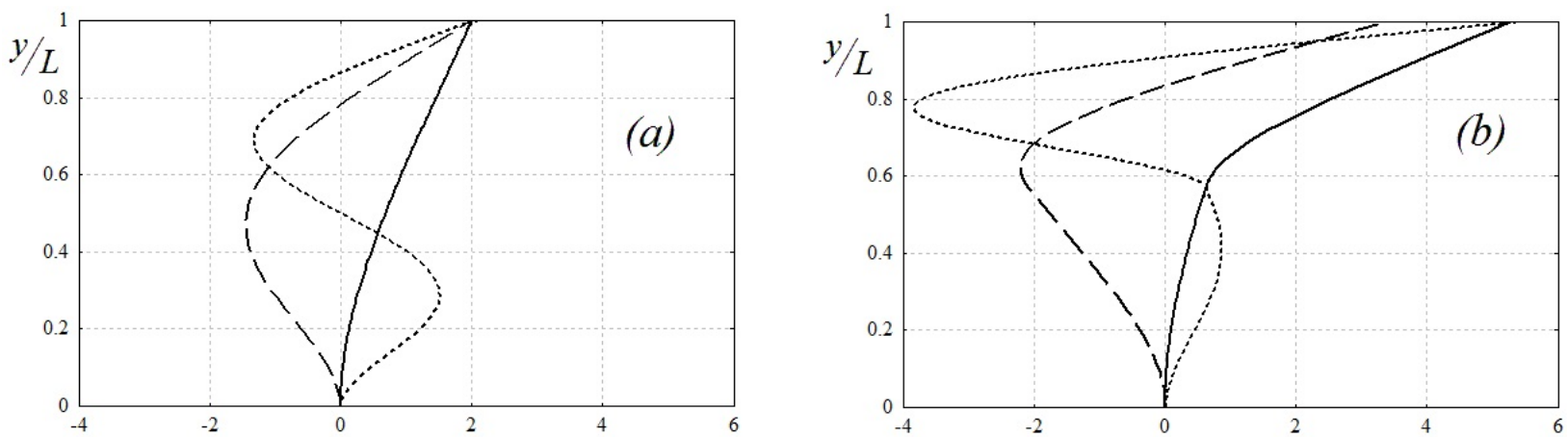

Fig. 7 Shapes of the first three normal modes (a) constant plate thickness, $\delta=1$, (b) NO96 tongue with $\delta=4 / 7$ and $\mu=5 / 19$. Solid lines are for the first modes, dashed lines are for the second and the dotted lines are for the third modes.

The problem (12)-(20) is coupled. The hydrodynamic loads on the plate depend on the velocity of the plate deflection through the body boundary condition (16).

The jet impact problem is solved by the normal-mode method ${ }^{19-22}$. The plate deflection $w(y, t)$ within this method is sought in the form

$$
w(y, t)=\sum_{n=1}^{\infty} a_{n}(t) \psi_{n}(y)
$$

where $a_{n}(t)$ are unknown principal coordinates of the plate deflection and $\psi_{n}(y)$ are the non-trivial solutions of the homogeneous boundary-value problem

$$
\begin{gathered}
\frac{\mathrm{d}^{2}}{\mathrm{~d} y^{2}}\left(h^{3}(y) \frac{\mathrm{d}^{2} \psi_{n}}{\mathrm{~d} y^{2}}\right)=\lambda_{n}^{4} h(y) \psi_{n}(y) \quad(0<y<1), \\
\psi_{n}(0)=\frac{\mathrm{d} \psi_{n}}{\mathrm{~d} y}(0)=0, \quad \frac{\mathrm{d}^{2} \psi_{n}}{\mathrm{~d} y^{2}}(1)=\frac{\mathrm{d}^{3} \psi_{n}}{\mathrm{~d} y^{3}}(1)=0,
\end{gathered}
$$

and $\lambda_{n}$ are the corresponding eigenvalues. Moreover, the eigenfunctions $\psi_{n}(y)$ satisfy the orthogonality condition

$$
\int_{0}^{1} h(y) \psi_{n}(y) \psi_{m}(y) \mathrm{d} y=\delta_{n m},
$$

where $\delta_{n m}=0$ for $n \neq m$ and $\delta_{n n}=1$ (see Appendix B). In the case of piecewise constant thickness of the plate, the modes $\psi_{n}(y)$ and their first derivatives are continuous at $y=\delta$. The second and third derivatives at this point are related by $\mu^{3} \psi_{n}^{\prime \prime}(\delta+0)=\psi_{n}^{\prime \prime}(\delta-0)$ and $\mu^{3} \psi_{n}^{\prime \prime \prime}(\delta+0)=\psi_{n}^{\prime \prime \prime}(\delta-0)$. The first three normal modes are shown in Figure 7 for the plate of constant thickness and the NO96 tongue.

Equations (14)-(17) and the series (21) lead to the following decomposition of the velocity potential

$$
\varphi(x, y, t)=\varphi_{0}(x, y) \chi(t)-\sum_{n=1}^{\infty} \dot{a}_{n}(t) \varphi_{n}(x, y),
$$

where $\varphi_{n}(x, y), n \geq 0$, are solutions of the boundary problem

$$
\nabla^{2} \varphi_{n}=0 \quad(x>0, \quad 0<y<\gamma)
$$




$$
\begin{array}{rlll}
\varphi_{n, y}(x, 0)=0, \quad \varphi_{n}(x, \gamma)=0 & (x>0), & \varphi_{n}(0, y)=0 \quad(1<y<\gamma), \\
\varphi_{n, x}(0, y)=\psi_{n}(y) \quad(0<y<1), & \varphi_{n} \rightarrow 0 \quad(x \rightarrow \infty),
\end{array}
$$

with $\psi_{0}(y)=1$. The mixed boundary value problem (26) is solved in Appendix A by the method of separating variables for $\gamma<1$ and by the theory of analytical functions for $\gamma>1$ with analytic treatment of the flow velocity singularity at the point $x=0, y=1$, where the boundary condition changes its type.

Substituting (21) and (25) in the beam equation (12), multiplying both sides of the equation by $\psi_{m}(y), m>1$, integrating both sides of the equation in $y$ from $y=0$ to $y=1$, and using the orthogonality condition (24), we arrive at the infinite system of ordinary differential equations with respect to the principal coordinates of the plate deflection,

$$
\begin{gathered}
\ddot{a}_{m}+\lambda_{m}^{4} a_{m}=\alpha \int_{0}^{1} p(0, y, t) \psi_{m}(y) \mathrm{d} y \\
\int_{0}^{1} p(0, y, t) \psi_{m}(y) \mathrm{d} y=-\frac{\mathrm{d}}{\mathrm{d} t} \int_{0}^{1} \varphi(0, y, t) \psi_{m}(y) \mathrm{d} y=-\frac{\mathrm{d}}{\mathrm{d} t}\left(-f_{m} \chi(t)+\sum_{n=1}^{\infty} M_{n m} \dot{a}_{n}\right),
\end{gathered}
$$

where

$$
f_{m}=-\int_{0}^{1} \varphi_{0}(0, y) \psi_{m}(y) \mathrm{d} y, \quad M_{n m}=-\int_{0}^{1} \varphi_{n}(0, y) \psi_{m}(y) \mathrm{d} y .
$$

Green's second identity gives that the matrix $M$ with the elements $M_{n m}$ is symmetric. The system (27) can be written in the form

$$
\frac{\mathrm{d}}{\mathrm{d} t}\left(\dot{a}_{m}+\alpha \sum_{n=1}^{\infty} M_{n m} \dot{a}_{n}-\alpha f_{m} \chi(t)\right)+\lambda_{m}^{4} a_{m}=0
$$

and finally in the matrix form

$$
\frac{\mathrm{d} \vec{a}}{\mathrm{~d} t}=(I+\alpha M)^{-1}(\vec{b}+\alpha \vec{f} \chi(t)), \quad \frac{\mathrm{d} \vec{b}}{\mathrm{~d} t}=D \vec{a},
$$

where $\vec{a}=\left(a_{1}, a_{2}, a_{3}, \ldots .\right)^{T}, \vec{b}=\left(b_{1}, b_{2}, b_{3}, \ldots .\right)^{T}, \vec{f}=\left(f_{1}, f_{2}, f_{3}, \ldots .\right)^{T}, I$ is the unit matrix and $D$ is the diagonal matrix, $D=\operatorname{diag}\left\{-\lambda_{1}^{4},-\lambda_{2}^{4}, \ldots\right\}$. The initial conditions for the system (28) are

$$
\vec{a}(+0)=0, \quad \vec{b}(+0)=0 .
$$

Note that the velocity of the plate at the impact time instant is not equal to zero,

$$
\frac{\mathrm{d} \vec{a}}{\mathrm{~d} t}(+0)=\alpha(I+\alpha M)^{-1} \vec{f} \neq 0 .
$$

Within the Kelvin-Voigt damping model, see equation (19), the system of differential equations (28) is changed to

$$
\frac{\mathrm{d} \vec{a}}{\mathrm{~d} t}=(I+\alpha M)^{-1}\left(\vec{b}+\alpha \vec{f} \chi(t)+\tau_{D} D \vec{a}\right), \quad \frac{\mathrm{d} \vec{b}}{\mathrm{~d} t}=D \vec{a} .
$$

In order to use the condition of the plate damage (5), it is convenient to introduce the nondimensional deflection $\langle w\rangle(y, t)$ averaged over the time interval of duration $\tau$, see condition $(5)$,

$$
<w>(y, t)=\frac{1}{\tau} \int_{t-\tau / 2}^{t+\tau / 2} w\left(y, t_{0}\right) \mathrm{d} t_{0} .
$$


Then the condition (5) reads

$$
\max _{0<t<T_{f} / T} \max _{0<y<1}|<\sigma>(y, t)|<\sigma_{Y} /\left(\sqrt{3} \rho_{p} c_{p} V\right),
$$

where the averaged in time deflection, $\langle w\rangle(y, t)$, and the averaged in time stress, $\langle\sigma\rangle(y, t)$, are related by (20). The averaged principal coordinates $\left\langle a_{n}\right\rangle(t)$ satisfy the system (28) without damping or (30) with damping, where the function $\chi(t)$ is changed to $\hat{\chi}(t)$ with $\hat{\chi}(t)=0$, where $t<-\tau / 2, \hat{\chi}(t)=t / \tau+1 / 2$, where $-\tau / 2<t<\tau / 2$, and $\hat{\chi}(t)=1$, where $t>\tau / 2$. It is seen that the averaging procedure is equivalent to smoothing the impact velocity by introducing the initial interval of short duration $\tau$, during which the velocity increases linearly from zero to the designed constant value. The initial conditions for the averaged deflection $\langle w\rangle(y, t)$, where $t>\tau / 2$, are

$$
<\vec{a}>(-\tau / 2)=0, \quad<\vec{b}>(-\tau / 2)=0 .
$$

Denoting the left-hand side in (32) by $C\left(\tau, \tau_{D}, \gamma\right)$, we conclude that the material of the plate behaves plastically during the jet impact on it, when the speed of the jet is greater than

$$
V>\frac{V_{p}}{C\left(\tau, \tau_{D}, \gamma\right)}, \quad V_{p}=\frac{\sigma_{Y}}{\sqrt{3} \rho_{p} c_{p}} .
$$

For the NO96 tongue, we have $V_{p} \approx 7.5 \mathrm{~m} / \mathrm{s}$. The critical velocity of the jet, $V_{p} / C$, starting from which the plate behaves plastically during impact, can be estimated once we know the maximum stress in the plate, $C\left(\tau, \tau_{D}, \gamma\right)$, which depends on the non-dimensional jet thickness $\gamma$, nondimensional retardation time $\tau$ and the non-dimensional stress relaxation time $\tau_{D}$.

\section{Numerical results and their discussion}

The linearized problem of jet impact onto clamped elastic plate is studied numerically for the NO96 tongue. Calculations of the integrals $f_{m}$ and $M_{n m}$ in (27) are detailed in the Appendix A. The infinite systems (28) and (30), as well as the corresponding systems for the averaged deflection with $\chi(t)$ changed to $\hat{\chi}(t)$, are truncated and integrated by the Runge-Kutta method of fourth order with corresponding initial conditions. Calculations are performed with $N_{\text {mod }}=3,5,10,15$ elastic modes in (21). The step of integration $\Delta t$ is equal to $1 / 10$ of the non-dimensional period of the highest retained mode with number $N_{\text {mod }}$. The stress distributions along the plate, the evolutions of bending stresses at critical points of the plate and the maximum non-dimensional stress $C\left(\tau, \tau_{D}, \gamma\right)$ as a function of the non-dimensional jet thickness $\gamma$, non-dimensional retardation time $\tau$ and the non-dimensional stress relaxation time $\tau_{D}$ are studied for the NO96 tongue and LNG jet. The density of the jet fluid appears only in the parameter $\alpha$, see equation (12). Changing the LNG to water, we need to change $\alpha$ from 1.1 to 2.36 .

The non-dimensional stress $\tilde{\sigma}(\delta+0, \tilde{t})$ at the place, where the tongue thickness abruptly changes, as a function of time for $\gamma=\frac{1}{2}$ and $\gamma=2$, is shown in figures 8 (a), left and right respectively. The stress has been computed with $N_{\text {mod }}=3$ and 10 in the interval $0<\tilde{t}<1.75$. The figures 8(b) depict the bending stress at the lowest point of the plate, $\tilde{y}=0$, as a function of time $\tilde{t}$. It is seen that the stresses at $\tilde{y}=0$ are smaller than at $\tilde{y}=\delta+0$ for both values of $\gamma$. The stresses can be decomposed in the "slow-varying" parts, which are represented by the lowest three modes, and the "high-frequency" perturbations. The stress $\tilde{\sigma}(\delta+0, \tilde{t})$ computed with three modes peaks at $\tilde{t} \approx 0.55$ for both values of $\gamma$. The stresses and deflections along the plate at $\tilde{t}=0.55$ are shown in figures $8(\mathrm{c})$ and $8(\mathrm{~d})$ respectively for $\gamma=\frac{1}{2}$ and $\gamma=2$. It is seen that the distributions 
$\gamma=0.5$
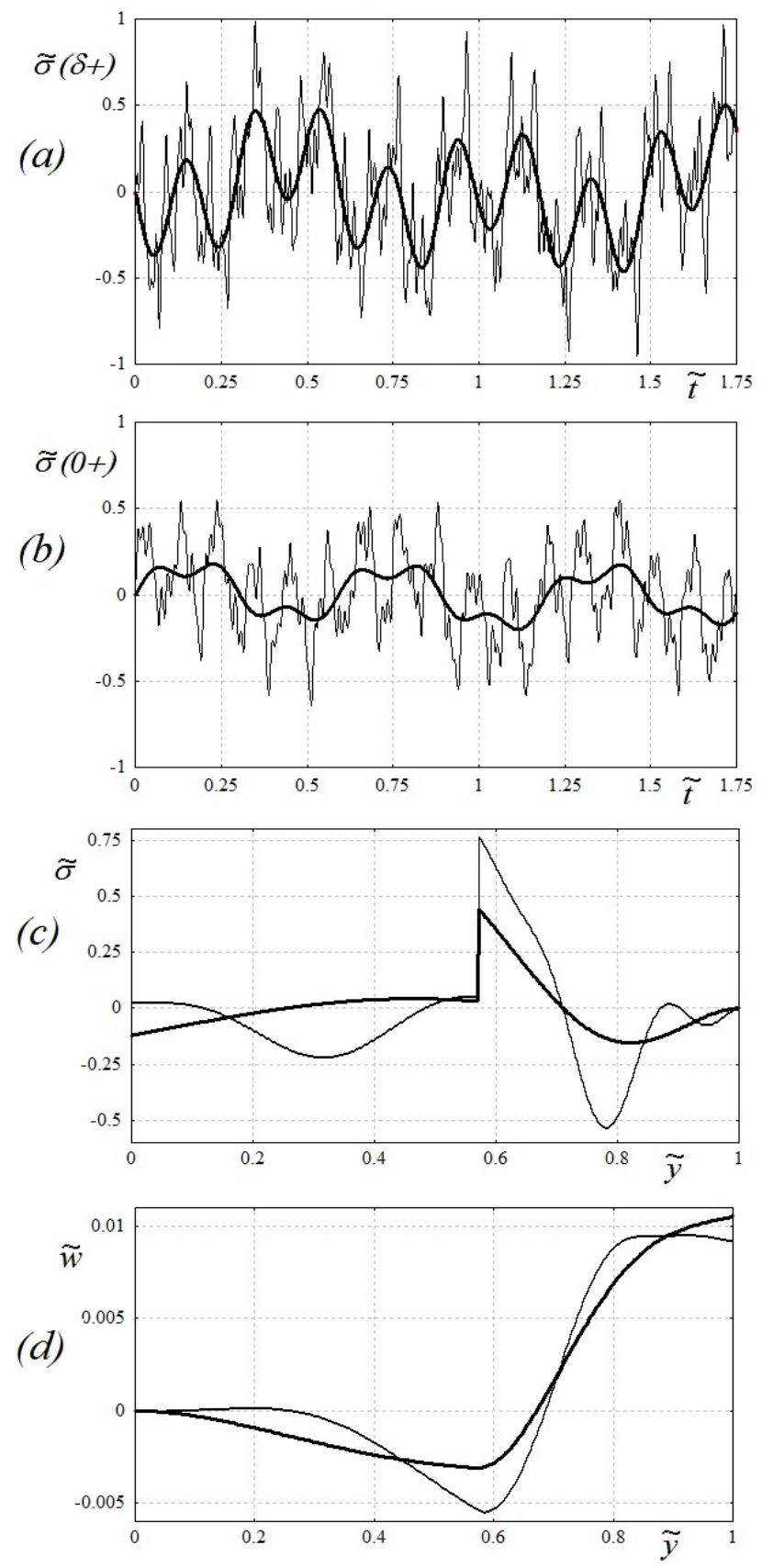

$\gamma=2$
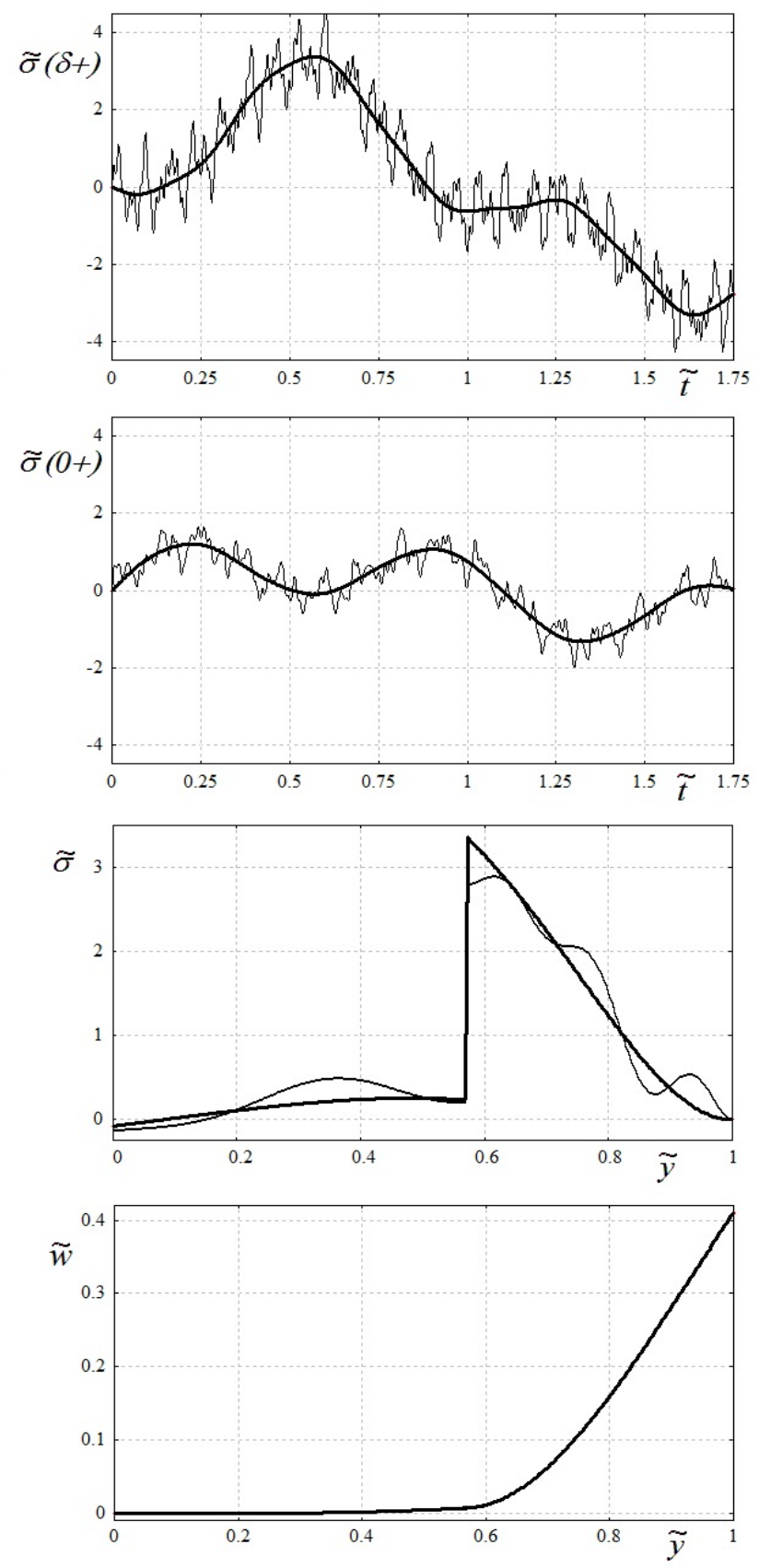

Fig. 8 The non-dimensional bending stresses and deflections calculated for $\tau=0$ and $\tau_{D}=0$ with three (thick solid lines) and ten (thin solid lines) modes retained in the series representation of the plate deflection (21) for the non-dimensional jet thickness $\gamma=\frac{1}{2}$ (left) and $\gamma=2$ (right).

(a) The non-dimensional bending stress $\tilde{\sigma}(\delta+0, t)$ at the boundary between the raised edge and the tongue, $\tilde{y}=\delta+0$, as a function of the non-dimensional time $\tilde{t}$,

(b) The non-dimensional bending stress $\tilde{\sigma}(0, t)$ at the clamped end of the plate $\tilde{y}=0$, as a function of the non-dimensional time $\tilde{t}$,

(c) The distribution of the bending stress along the tongue at $\tilde{t}=0.55$ computed with three and ten elastic modes,

(d) The plate deflection along the tongue at $\tilde{t}=0.55$ computed with three and ten elastic modes. 
along the plate are rather smooth in contrast to the stress evolution in time. The contributions of the higher modes are stronger for narrow jets, see left figures $8(\mathrm{c})$ and $8(\mathrm{~d})$ for $\gamma=\frac{1}{2}$, than for thick jets. However, even for $\gamma>1$, the convergence in terms of the number of modes is observed only for the deflections, see right figure $8(\mathrm{~d})$, but not for the stresses. The stresses become bigger with the increase of the number of modes. It is clear does the series for bending stress obtained from (21) converge theoretically but it does not converge practically. Therefore, the values of the retardation time $T_{r}$ and/or stress relaxation time $\tau_{D}$ are important to estimate the bending stresses by the present simplified model.

The present model of jet impact onto elastic plate is simplified in terms of the impact conditions. In reality, the jet front is not parallel to the plate ${ }^{23}$ at impact instant (figure 3(a)), compressibility ${ }^{24}$ (figure $3(\mathrm{~b})$ ) and aeration ${ }^{12,13}$ (figure $3(\mathrm{c})$ ) of the fluid in the impact region matter, as well as the presence of the air ${ }^{10,11,22}$ in between the plate and the approaching jet front (figure $3(\mathrm{~d})$ ). These effects make the hydrodynamic loading on the plate to be gradual in time and can be described by using the concept of retardation time, $T_{r}$. For example, for the NO96 tongue and the jet of compressible water with the sound speed in water $c_{0}=1500 \mathrm{~m} / \mathrm{s}$, the retardation time can be estimated as the time needed for the sound wave to travel from the upper edge of the plate to the bottom, $T_{r}=L / c_{0} \approx 2.5 \times 10^{-5} \mathrm{~s}$, which gives the non-dimensional retardation time $\tau=T_{r} / T \approx 0.05$. The effect of the retardation time on the bending stress at $\tilde{y}=\delta+0$ is shown in figure 9 for $\gamma=\frac{1}{2}$ (left) and $\gamma=2$ (right) with the non-dimensions retardation time $\tau=0.075$ and $\tau=0.05$. The figure 9 shows that even small values of the non-dimensional retardation time significantly reduce the contributions of the higher modes to the bending stresses. In order to explain such a significant effect of retardation time on bending stresses, we compute the maximum bending stresses by (32) for different values of $\tau$. The figure 10 shows that the maximum stress in the NO96 tongue does not vary significantly for $\tau>0.05$. The results of calculations are shown in figure 10a for three, five and ten modes. It is clear that the maximum stress is weakly dependent on the number of modes for $\tau>0.05$. This fact is explained in figure 10b, where the non-dimensional periods, $T_{n}$, of the dry elastic modes, $\psi_{n}(y)$, governed by equations $(22)$ and (23), are depicted. The periods $T_{n}$ are shown by diamonds for the NO96 tongue $(\mu<1)$ and by crosses for the equivalent tongue of constant thickness $(\mu=1)$ for $n \geq 4$. The first four non-dimensional periods of the NO96 tongue are: $T_{1} \approx 1.51, T_{2} \approx 0.59, T_{3} \approx 0.19, T_{4} \approx 0.1$, and the periods of the tongue with constant thickness, which is the same tongue as the NO96 one but with $\mu=1$, are: $T_{1} \approx 1.79, T_{2} \approx 0.29$, $T_{3} \approx 0.1, T_{4} \approx 0.05$. The figure $10 \mathrm{~b}$ shows that the retardation time $\tau=0.05$ is greater than the periods of elastic modes starting from $n=5$ for the NO96 tongue. Then the modes starting from the fifth one can be excluded from calculations for such a value of the retardation time. However, for the tongue of constant thickness (crosses in figure 10b) the required number of modes to retain is six. Therefore, higher modes are stronger pronounced for the tongue of constant thickness than for the tongue of variable thickness.

The effect of structural damping on the evolution of the bending stresses is depicted in figure 11. The non-dimensional relaxation time $\tau_{D}$ is equal to 0.01 and 0.001 in these calculations with ten elastic modes. It is clear that structural damping reduces the contributions of the higher modes in the bending stresses but also reduces the magnitudes of the stresses with time. On the other hand, reduction of the first peak of the stress evolution is not significant. We can say that small structural damping does not affect the maximum stress in the elastic plate during jet impact on it. 

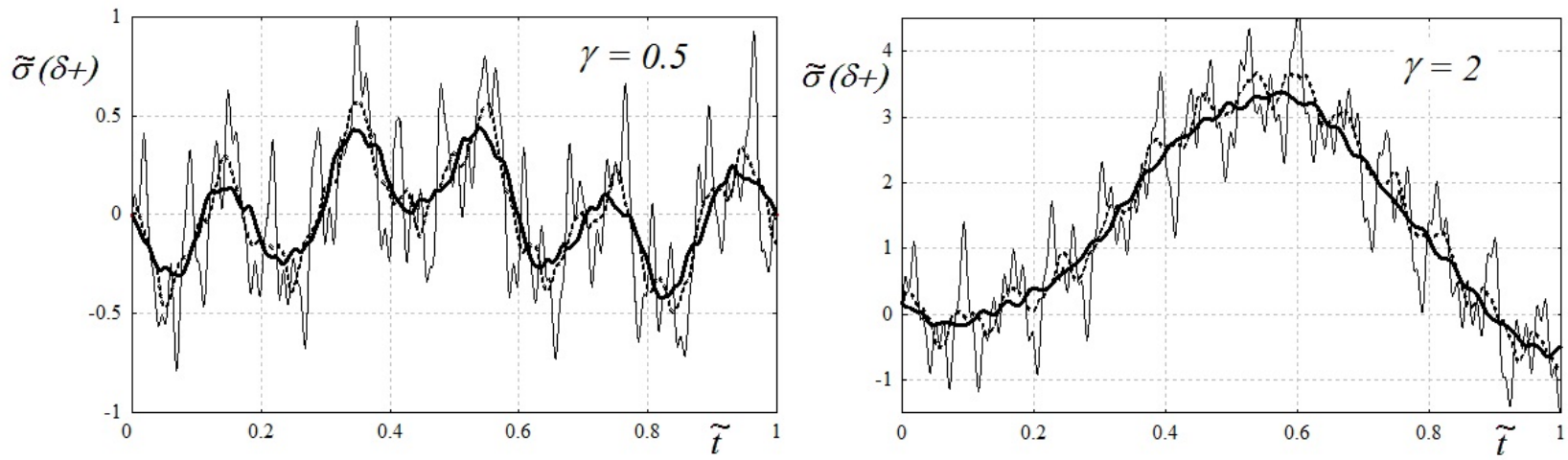

Fig. 9 The bending stress at $\tilde{y}=\delta+0$ computed with ten elastic modes and $\tau=0$ (thin line), $\tau=0.075$ (thick line) and $\tau=0.05$ (dotted line).
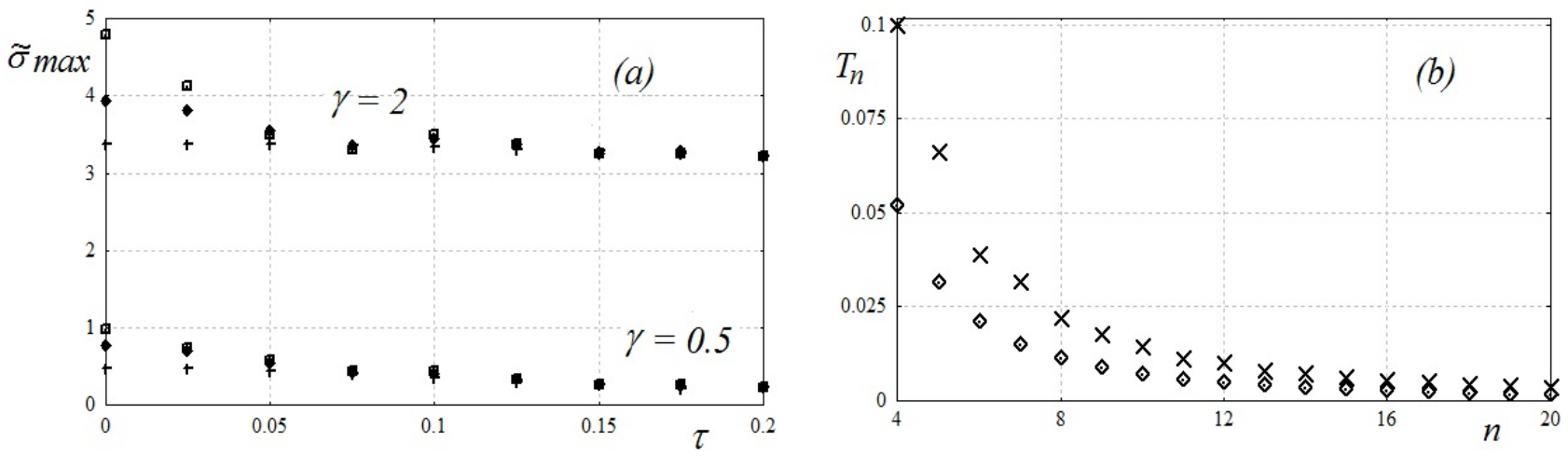

Fig. 10 (a) The maximum bending stress given by the left-hand side of equation (32) as a function of the non-dimensional retardation time $\tau$ for the non-dimensional jet thickness $\gamma=\frac{1}{2}$ and $\gamma=2$.

The stresses are calculated with three (crosses), five (diamonds) and ten (squares) modes.

(b) The non-dimensional periods, $T_{n}$, of the dry elastic modes, $\psi_{n}(y)$, as functions of the number $n$ for the NO96 tongue (diamonds) and the same tongue but of constant thickness, $\mu=1$, (crosses).
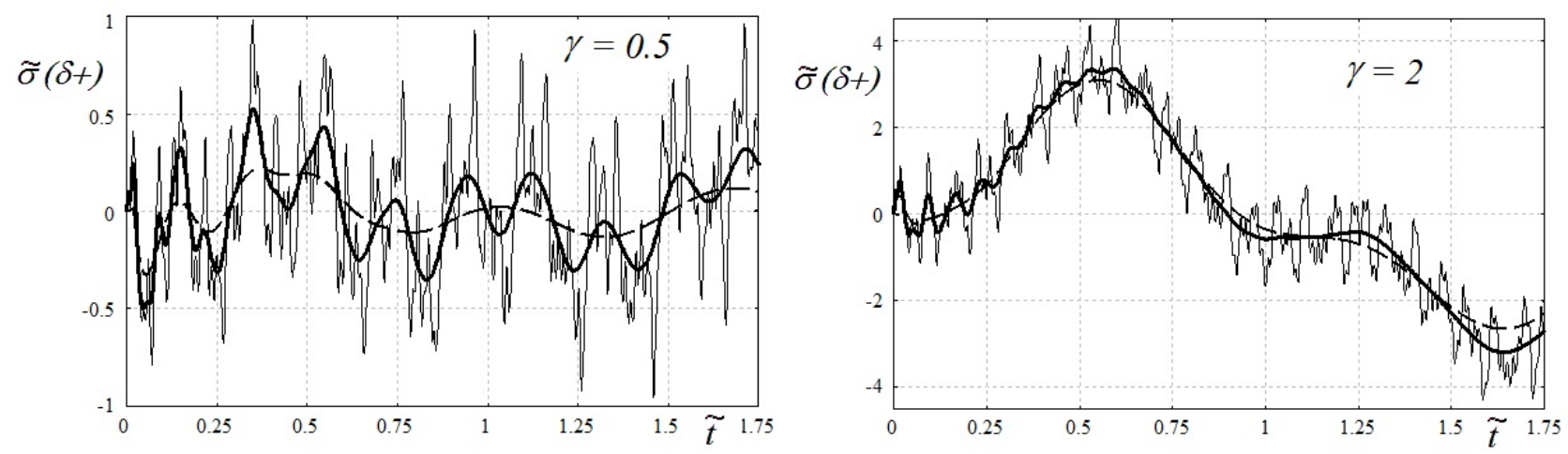

Fig. 11 Time evolutions of the non-dimensional stress at the point $\tilde{y}=\delta+0$ calculated with ten modes without effects of damping and retardation (thin lines) and with account for damping with $\tau_{D}=0.001$ (thick lines) and $\tau_{D}=0.01$ (dashed lines). 


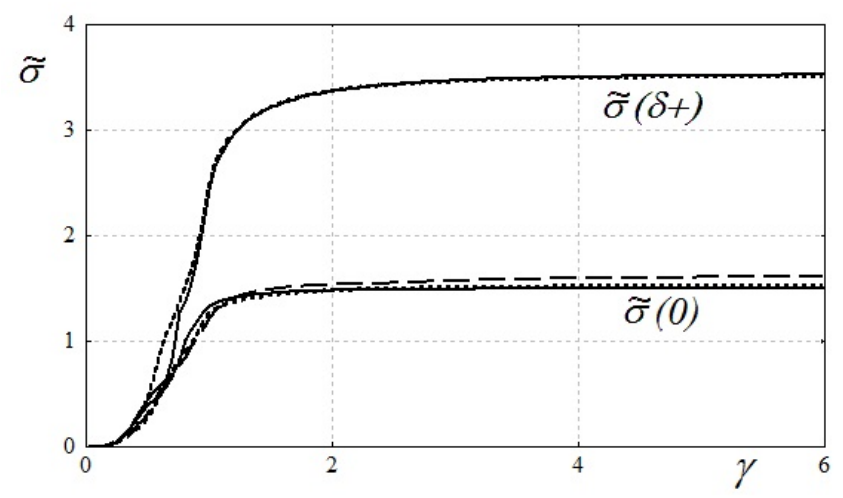

Fig. 12 The maximum values of the non-dimensional bending stresses in the NO96 tongue at $\tilde{y}=0$ and $\tilde{y}=\delta+0$ in the time interval $0<\tilde{t}<2$ as functions of the non-dimensional jet thickness $\gamma$ calculated with four modes and $\tau=0$ (solid lines), with ten modes and $\tau=0.075$ (dotted lines), and for the NO96 tongue of constant thickness, $\mu=1$, with ten modes and $\tau=0.075$ (dashed line). Note that the maximum stress given by the left-hand side of (5) is achieved at $\tilde{y}=\delta+0$ for the NO96 tongue and at $\tilde{y}=0$ for the equivalent plate of constant thickness.

The maximum non-dimensional stresses in the NO96 tongue of variable thickness and the equivalent tongue of the constant thickness $(\mu=1)$ are shown in figure 12 as functions of the non-dimensional jet thickness $\gamma$. The maximum averaged stress is defined by the left-hand side of (5). In calculations, $T_{f}=2 T$, the step along the plate is smaller than 0.05 in both intervals $0 \leq \tilde{y} \leq \delta$ and $\delta \leq \tilde{y}<1$, the step in time is 0.01 in the non-dimensional variables. Note that the bending stress is discontinuous at $\tilde{y}=\delta$, see figure $8 \mathrm{c}$. The calculations provide that the maximum bending stress is achieved at $\tilde{y}=\delta+0$ for any thickness of the jet. Actually the stress maximum at $\tilde{y}=\delta+0$ during the time interval $0<\tilde{t}<2$ is shown in this figure. Increasing the time interval does not change the resulting value of the maximum stress. The calculations were performed with four modes without averaging and damping, $\tau=0$ and $\tau_{D}=0$ (solid line), and with ten modes and averaging with $\tau=0.075$ but without damping (dotted line). In addition, the maximum stress at $\tilde{y}=0$ during the same time interval is also shown in the figure. It is seen that both maximum stresses, $\tilde{\sigma}(\delta+0)$ and $\tilde{\sigma}(0)$, are weakly dependent on $\gamma$ for $\gamma>2$. The absolute non-dimensional maximum of the stress for any thickness of the jet is equal approximately to 3.5 and it is approached for large $\gamma$. We obtained in particular, $\tilde{\sigma}(\delta+0)=3.4927$ for $\gamma=4$ and $\tilde{\sigma}(\delta+0)=3.5138$ for $\gamma=6$ with ten modes and $\tau=0.075$. The dashed line in figure 12 is for the maximum bending stress in the tongue of constant thickness. This line suggests that the maximum stress at $\tilde{y}=0$ is weakly dependent on the variation of the plate thickness but depends strongly on the plate length.

Inequality (33) provides that the plastic deformations of the NO96 tongue start at the place, where the tongue changes its thickness, when the speed of the LNG jet impact is greater than 2.15 $\mathrm{m} / \mathrm{s}$. Plastic deformations occur at $y=0$ when the speed of the jet impact is greater than $5 \mathrm{~m} / \mathrm{s}$.

The developed model of water impact is applied to the $2 \mathrm{D}$ problem of a dam-break flow in the presence of an obstacle. This problem was studied using an SPH projection method by Rafiee and Thiagarajan ${ }^{25}$ for a hypoelastic baffle with density $\rho_{p}=2500 \mathrm{~kg} / \mathrm{m}^{3}$, Young modulus $E=$ $1.0 \times 10^{6} \mathrm{~Pa}$, height $8 \mathrm{~cm}$ and thickness $h_{1}=1.2 \mathrm{~cm}$. Initially the water column was $L=14.6 \mathrm{~cm}$ wide and $2 L=29.2 \mathrm{~cm}$ high. The baffle was placed at distance $L$ to the right of the water column. Air was neglected in the simulations. The gravity was switched on at $t=0$. To estimate the impact conditions, we assume that the initial water column, $14.6 \times 29.2 \mathrm{~cm}$, occupies the region between the wall on the left of the column and the obstacle on the right and is of constant depth, $29.2 \times 14.6 \mathrm{~cm}$ at the impact instant. The initial potential energy of the water column, $2 \rho g L^{3}$, is 
equal to the sum of the potential energy of the water at the impact instant, $\rho g L^{3}$, and the kinetic energy of the impact flow. The flow velocity at the impact instant can be estimated as a constant $V_{i m p}$, then the kinetic energy of the flow at the impact instant $\frac{1}{2} \rho V_{i m p}^{2} \cdot 2 L \cdot L=2 \rho g L^{3}-\rho g L^{3}$ provides $V_{i m p}=\sqrt{g L} \approx 1.2 \mathrm{~m} / \mathrm{s}$. If the flow velocity is approximated by a linear function of the distance $x$ from the wall to the left from the obstacle, $V=V_{i m p} x /(2 L)$, then the kinetic energy is $\frac{1}{3} \rho L^{2} V_{i m p}^{2}$ and we find $V_{i m p}=\sqrt{3 g L} \approx 2 \mathrm{~m} / \mathrm{s}$. The calculation of the deflection by present model is performed with $V_{i m p}=1.5 \mathrm{~m} / \mathrm{s}$. This value of the impact speed is in between two estimates obtained above. Figure 9 in the paper by Rafiee and Thiagarajan ${ }^{25}$ shows the deformation of the elastic baffle and the fluid flow at several time instants. Using this figure, we estimate the retardation time $T_{r}$ as $0.1 \mathrm{sec}$. This is the time needed for the baffle to be completely wetted in the numerical simulations. The time history of the displacement of the upper left corner of the baffle computed by the model of this paper is compared with the results by Rafiee and Thiagarajan ${ }^{25}$ and three other available numerical results, see Figure $10^{25}$. The numerical results provide the maximum displacement in the range $4.1-4.8 \mathrm{~cm}$ achieved approximately $0.1 \mathrm{sec}$ after the beginning of the baffle interaction with the fluid. Our model predict the maximum deflection of $4.75 \mathrm{~cm}$ at $t=0.1 \mathrm{sec}$ which well correspond to the results of the numerical simulations by others. Note that the considered conditions of the fluid-structure interaction are rather far from the jet impact conditions of the present paper. However, both numerical and theoretical results show that the maximum deflection of the baffle is achieved shortly after the beginning of the interaction. Later on the baffle oscillates with quite different displacement histories predicted by different numerical methods.

\section{Conclusion}

The bending stresses in a clamped elastic plate impacted by a liquid jet have been estimated within the two-dimensional coupled model of hydroelasticity. The configuration of the problem and the impact conditions are simplified within the model. It was shown that the stresses during the initial impact stage are higher than stresses in the plate placed in the equivalent uniform flow. The problem of jet impact has been solved by the normal mode method during the initial stage. The present model over-predicts the contributions of the higher modes. The series for the bending stress converges but not absolutely. This follows from the analysis of equation (27) and the decay of the added-mass effects with the mode number.

In reality, the jet front is not parallel to the plate at impact instant, compressibility and aeration of the fluid in the impact region, as well as the presence of the air in between the plate and the approaching jet front, matter. These effects make the hydrodynamic loading on the plate to be gradual in time and can be described, in terms of the maximum bending stress in the plate, by using the concept of retardation time, which characterizes the duration of the early transient stage. In the present study, this duration was assumed to be smaller than fourth natural period of the elastic plate vibration in air. In practical situations, the retardation time can be even larger than the period of the lowest elastic mode. Then the present solution provides an upper estimate of the maximum bending stress in the plate. For some effects the retardation time can be estimated. The idea behind the concept of the retardation time is that the complex problem of fluid impact onto an elastic plate in practical situations can be split into two: (1) the problem of jet impact onto a vertical elastic plate with the jet front being vertical and the retardation time given, (2) estimation of the retardation time for a particular situation. The concept of the retardation time is also related to the condition of the plate failure by the jet impact. In the present model, it is required that the bending stress in the plate should stay above the yield stress value for a certain 
time before plastic deformations start, see inequality (5).

The failure condition adopted in the present study does not imply that the NO96 tongue is completely broken after a single jet impact. The condition implies that the plastic deformations in the tongue start and the plate does not return after the impact to its initial position.

We conclude that, if plastic deformations did not start in a clamped elastic plate during the initial jet impact stage, they will not occur in the plate after the end of the impact stage when the flow around the plate is well developed. It was shown that the maximum stress in the plate is proportional to the jet thickness and is bounded from above by the stress value computed for the jet of infinite thickness.

Acknowledgment: The authors thank Dr. Laurent Brosset (Gaztransport \& Technigaz, France) for his guidance about the NO96 membrane system and its failure conditions. Third author acknowledges the support of the National Research Foundation of Korea (NRF) grant funded by the Korea Government (MEST) through GCRC-SOP.

\section{References}

${ }^{1}$ See http://www.gtt.fr/en/technologies-services/our-technologies/no96 for the description of the NO96 membrane system.

${ }^{2}$ O. Kimmoun, L. Brosset and G. Dupont, "Experimental study of wave impacts on a corrugated ceiling," Proc. ISOPE (2016).

${ }^{3}$ C. Lugni, A. Bardazzi, O. M. Faltinsen and G. Graziani, "Hydroelastic slamming response in the evolution of a flip-through event during shallow-liquid sloshing," Phys. Fluids 26 (3), 032108 (2014).

${ }^{4}$ G. Oger, P.M. Guilcher, E. Jacquin, L. Brosset, J.B. Deuff, and D. Le Touze, "Simulations of hydro-elastic impacts using a parallel SPH model", Proc. ISOPE (2009).

${ }^{5}$ L. Brosset, Z. Mravak, M. Kaminski, S. Collins and T. Finnigan, "Overview of Sloshel project," Proc. ISOPE (2009).

${ }^{6}$ W. Lafeber, H. Bogaert, and L. Brosset, "Elementary Loading Processes (ELP) involved in breaking wave impacts: findings from the Sloshel project," Proc. ISOPE (2012).

${ }^{7}$ S. Shafiei, B. W. Melville and A. Y. Shamseldin, "Experimental investigation of tsunami bore impact force and pressure on a square prism," Coastal Engineering 110, 1-16 (2016).

${ }^{8}$ Z. Wei, R.A. Dalrymple, A. Herault, G. Bilotta, E. Rustico and H. Yeh, "SPH modeling of dynamic impact of tsunami bore on bridge piers," Coastal Engineering 104, 26-42 (2015).

${ }^{9}$ H. Yeh, "Tsunami bore runup," In: Tsunami Hazard. Springer, Netherlands, 209-220 (1991).

${ }^{10}$ D. H. Peregrine, " Water-wave impact on walls," Ann. Rev. Fluid Mech. 35 (1), 23-43 (2003). 
${ }^{11}$ D. H. Peregrine and L. Thais, "The effect of entrained air in violent water wave impacts," J. Fluid Mech. 325, 377-398 (1996).

${ }^{12}$ A.A. Korobkin, "Two-dimensional problem of the impact of a vertical wall on a layer of a partially aerated liquid," J Applied Mech. Tech. Phys. 47 (5), 643-653 (2006).

${ }^{13}$ A. Iafrati and A.A. Korobkin, "Breaking wave impact onto vertical wall," Proc. 4th Int. Conf. Hydroelas. Mar. Tech., Wuxi, China, 139-148 (2006).

14 Y.V. Petrov and A.A. Utkin, "Dependence of the dynamic strength on loading rate," Materials Science, 25(2), 153-156 (1989).

15 V.V. Novozhilov, "A necessary and sufficient criterion of brittle stability (Elastic bodies brittle stability weakened by cut, developing criterion for determining critical cut length)," PRIKLADNAIA MATEMATIKA I MEKHANIKA, 33, 212-222 (1969).

${ }^{16}$ M.I. Gurevich, "The Theory of Jets in an Ideal Fluid": International Series of Monographs in Pure and Applied Mathematics (Vol. 93). Elsevier, (2014).

${ }^{17}$ G. Birkhoff, M. Plesset, and N. Simmons, "Wall Effects in Cavity Flow - I," Quarterly of Applied Mathematics 8(2), 151-168 (1950).

${ }^{18}$ G.E. Mase, "Theory and Problem of Continuum Mechanics," Schaum's OutlineSeries, McGraw-Hill Book Company, 223 (1970).

${ }^{19}$ A.A. Korobkin, T. I. Khabakhpasheva and G. X. Wu, "Coupled hydrodynamic and structural analysis of compressible jet impact onto elastic panels," Journal of Fluids and Structures 24(7), 1021-1041 (2008).

${ }^{20}$ A.A. Korobkin, "Wave impact on the center of an Euler beam," J Applied Mech. Tech. Phys. 39 (5), 770-781 (1998).

${ }^{21}$ A.A. Korobkin and T.I. Khabakhpasheva, "Regular wave impact onto an elastic plate," Journal of Engineering Mathematics 55(1-4), 127-150 (2006).

${ }^{22}$ T.I. Khabakhpasheva, A. A. Korobkin and S. Malenica, "Fluid impact onto a corrugated panel with trapped gas cavity," Applied Ocean Research 39, 97-112 (2013).

${ }^{23}$ A.A. Korobkin, "Wagner theory of steep wave impact," In Proc. 23rd Int. Workshop on Water Waves and Floating Bodies, Jeju, Korea, 13-16 (2008).

${ }^{24}$ A.A. Korobkin and S. Malenica. "Steep wave impact onto elastic wall." In Proc. 22nd Int. Workshop on Water Waves and Floating Bodies, Plitvice, Croatia (2007).

${ }^{25}$ A. Rafiee and K.P. Thiagarajan, "An SPH projection method for simulating fluid-hypoelastic structure interaction," Computer Methods in Applied Mechanics and Engineering, 198(33), 2785-2795 (2009). 


\section{Nomenclature}

\begin{tabular}{|c|c|}
\hline$L$ & - the length of elastic plate \\
\hline$H$ & - the jet thicknes \\
\hline$V$ & - the jet speed \\
\hline$t$ & - the time \\
\hline$x$ & - the horizontal coordinate \\
\hline$y$ & - the vertical coordinate \\
\hline$h(y)$ & - the variable thickness of the elastic plate \\
\hline$\sigma(y, t)$ & - the stress \\
\hline$\sigma_{Y}$ & - $\quad$ the yield stress of the plate material \\
\hline$T_{r}$ & - the retardation time of the plate material \\
\hline$w(y, t)$ & - the plate deflection \\
\hline$\rho_{p}$ & - the density of the plate material \\
\hline$E$ & the Young module of the plate material \\
\hline$I(y)=h^{3}(y) / 12$ & - the moment of the inertia of the plate section \\
\hline$c_{p}=\left(E / \rho_{p}\right)^{\frac{1}{2}}$ & - the bar velocity of the plate material \\
\hline$Q(y, t)$ & - the shear force \\
\hline$M(y, t)$ & - the bending moment \\
\hline$\rho$ & - the density of the liquid \\
\hline$p(x, y, t)$ & - the hydrodynamic pressure \\
\hline$\Omega(t)$ & - the flow region \\
\hline$\varphi(x, y, t)$ & - the velocity potential \\
\hline$\vec{u}(x, y, t)$ & - the velocity field of the flow \\
\hline$\mu$ & - $\quad$ the ratio of the plate thickness \\
\hline$\delta$ & - the ratio of thick and total plate length \\
\hline$\varepsilon=V T / L$ & - the ratio of the fluid displacement \\
\hline$\tau_{D}$ & - the non-dimensional relaxation time \\
\hline$\gamma=H / L$ & - the non-dimensional jet thickness \\
\hline$\alpha=\rho L /\left(\rho_{p} h_{1}\right)$ & - the non-dimensional parameter \\
\hline$h_{1}$ & - thickness of the lower part of the plate \\
\hline$T$ & $-\quad$ the time scale \\
\hline$\psi_{n}(y)$ & $n$-th normal mode of the plate \\
\hline$\lambda_{n}$ & - the eigenvalue corresponding to the $n$-th normal mode \\
\hline$T_{n}$ & the non-dimensional natural period of the $n$-th mode \\
\hline$N_{\text {mod }}$ & - $\quad$ number of modes in numerical calculations \\
\hline$\varphi_{n}(x, y)$ & - $\quad$ solution of the boundary-value problem (26) \\
\hline$M$ & - the added-mass matrix with the elements $M_{n m}$ \\
\hline$\vec{a}(t)$ & the vector of the principle coordinates of the plate deflectio \\
\hline$\vec{b}(t)$ & - the auxiliary unknown vector \\
\hline$D$ & - the diagonal matrix \\
\hline$\vec{f}$ & - $\quad$ the vector defined in $(27)$ \\
\hline$<w>$ & - the averaged value over the time interval of duration $\tau$ \\
\hline$C\left(\tau, \tau_{D}, \gamma\right)$ & - $\quad$ the function defined by the left-hand side of $(32)$ \\
\hline$V_{p}$ & - $\quad$ the reference velocity defined by $(33)$ \\
\hline$\Delta t$ & - step of integration in time \\
\hline
\end{tabular}




\section{Appendix A: Added-mass matrix of jet impact problem}

The elements $M_{n m}$ of the added mass matrix $M$, where $n, m \geq 1$, are given by the integrals, see (27), along the elastic plate. It is known that the matrix $M$ is symmetric. The elements $f_{m}$ of the vector $\vec{f}$ are formally equal to $M_{0 m}$, where $\varphi_{0}(x, y)$ is the solution of the problem (26) with $\psi_{0}(y)=1$. The functions $\psi_{m}(y)$, where $m \geq 1$, are the normal modes of the elastic plate.

The integrals $M_{n m}$, where $m \geq 1$ and $n \geq 0$, can be reduced to infinite series for thin jets with $\gamma<1$, see figure 13(a). For $\gamma<1$, the potentials $\varphi_{n}(x, y)$ are given by the series

$$
\varphi_{n}(x, y)=\sum_{k=0}^{\infty} C_{n k} \cos \left(\mu_{k} y\right) e^{-\mu_{k} x}
$$

where $\mu_{k}=\pi(2 k+1) /(2 \gamma)$. The potentials (A1) satisfy all equations (26) except the body boundary condition, $\varphi_{n, x}(0, y)=\psi_{n}(y)$, where $0<y<\gamma$. Substituting (A1) in this boundary condition, multiplying both sides of this condition by $\cos \left(\mu_{p} y\right)$ and integrating in $y$ from $y=0$ to $y=\gamma$, we obtain the coefficients

$$
C_{n k}=-\frac{2}{\gamma \mu_{k}} Q_{n k}(\gamma), \quad Q_{n k}(\gamma)=\int_{0}^{\gamma} \psi_{n}(y) \cos \left(\mu_{k} y\right) \mathrm{d} y
$$

Substituting (A1) in the integrals $M_{n m}$, we find

$$
M_{n m}(\gamma)=-\int_{0}^{\gamma} \varphi_{n}(0, y) \psi_{m}(y) \mathrm{d} y=-\sum_{k=0}^{\infty} C_{n k} \int_{0}^{\gamma} \psi_{m}(y) \cos \left(\mu_{k} y\right) \mathrm{d} y=\frac{4}{\pi} \sum_{k=0}^{\infty} \frac{Q_{m k}(\gamma) Q_{n k}(\gamma)}{2 k+1}
$$

The integrals $Q_{n k}(\gamma)$ and the elements of the added-mass matrix $M_{n m}(\gamma)$ are pre-computed for a given $\gamma$. For $n=0$ and, correspondingly, $\psi_{0}(y)=1$, we obtain

$$
Q_{0 k}(\gamma)=\frac{2 \gamma(-1)^{k}}{\pi(2 k+1)}
$$

and

$$
f_{m}(\gamma)=\frac{8 \gamma}{\pi^{2}} \sum_{k=0}^{\infty} \frac{(-1)^{k} Q_{m k}(\gamma)}{(2 k+1)^{2}}
$$

For $\gamma>1$ formulae similar to (A3) and (A5) can be derived by using the conformal mapping $z=z(\zeta)$ of the unit semi-strip in the $\zeta$-plate onto the flow region in the $z$-plane, see figures $13(\mathrm{~b})$ and 13(c). Here $z=x+i y$ and $\zeta=\xi+i \eta$. The potentials $\varphi_{n}$, which solve the problems (26) for $n \geq 0$, written in the variables of the conformal mapping, $\xi$ and $\eta$, are denoted by $\Phi_{n}(\xi, \eta)$,

$$
\Phi_{n}(\xi, \eta)=\varphi_{n}(x(\xi, \eta), y(\xi, \eta)) .
$$

The potentials $\Phi_{n}(\xi, \eta)$ satisfy the same equation and the boundary conditions as in (26), see also figure 13(c), except the body boundary condition along the plate. This condition reads

$$
\Phi_{n, \xi}(0, \eta)=\psi_{n}[y(0, \eta)] y_{\eta}(0, \eta) \quad(\xi=0,0<\eta<1) .
$$



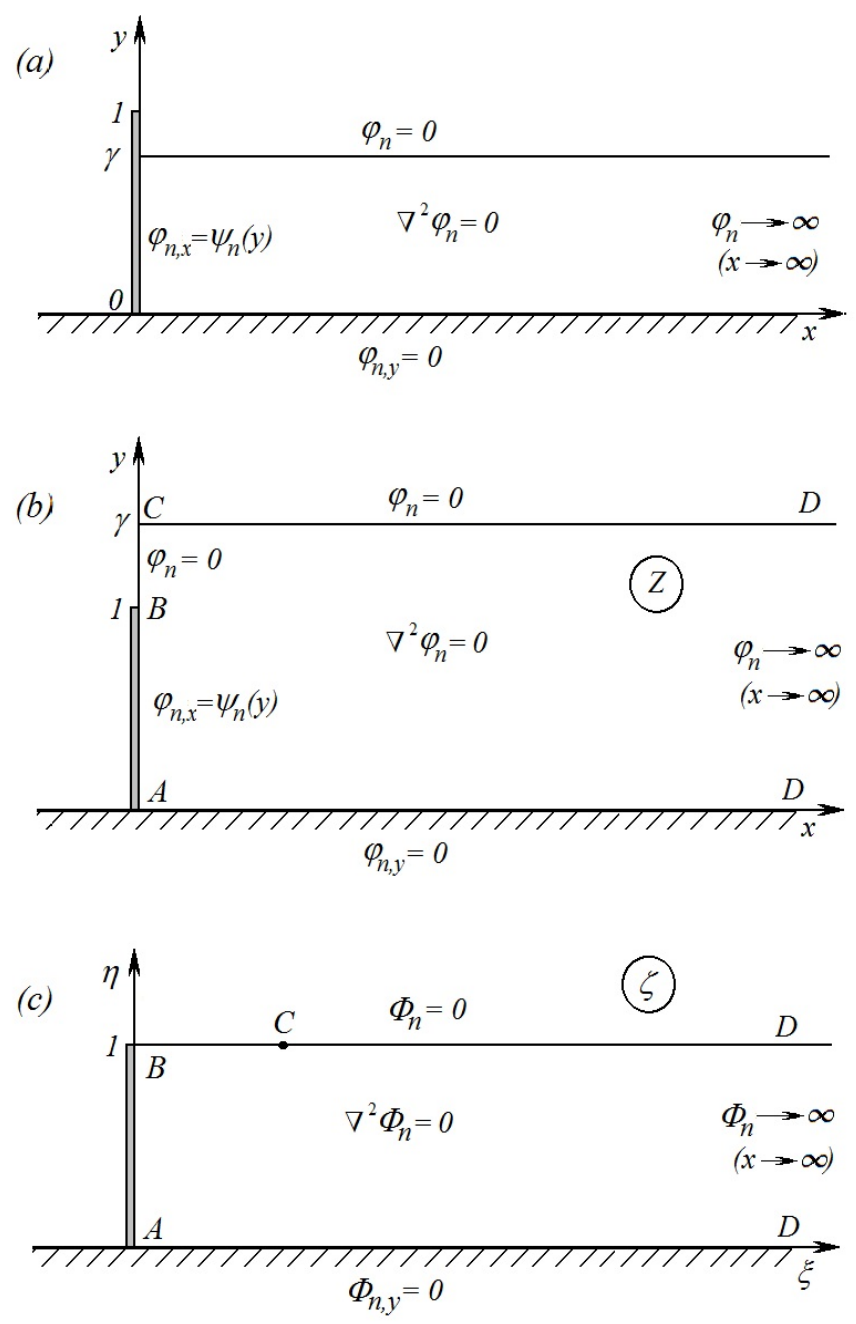

Fig. 13 The boundary problem (26) for (a) $\gamma<1$, (b) $\gamma>1$, and (c) the $\zeta$-plane of the conformal mapping $z=z(\zeta)$ with the correspondence of boundary points.

It is convenient to change the variable of integration $y$ in the integrals $M_{n m}$ for $\gamma>1$ to $\eta$. Then

$$
\begin{gathered}
M_{n m}(\gamma)=-\int_{0}^{1} \varphi_{n}(0, y) \psi_{m}(y) \mathrm{d} y= \\
-\int_{0}^{1} \varphi_{n}[0, y(0, \eta)] \psi_{m}[y(0, \eta)] y_{\eta}(0, \eta) \mathrm{d} \eta=-\int_{0}^{1} \Phi_{n}[0, \eta] \psi_{m}[y(0, \eta)] y_{\eta}(0, \eta) \mathrm{d} \eta .
\end{gathered}
$$

The potentials $\Phi_{n}(\xi, \eta)$ are sought in the form (A1),

$$
\Phi_{n}(\xi, \eta)=\sum_{k=0}^{\infty} C_{n k} \cos \left(\mu_{k} \eta\right) e^{-\mu_{k} \xi}, \quad \mu_{k}=\frac{\pi}{2}(2 k+1),
$$

where

$$
C_{n k}=-\frac{2}{\mu_{k}} Q_{n k}(\gamma), \quad Q_{n k}(\gamma)=\int_{0}^{1} \psi_{n}[y(0, \eta)] \cos \left(\mu_{k} \eta\right) y_{\eta}(0, \eta) \mathrm{d} \eta
$$


for $\gamma>1$. Substituting (A9) in (A8) and using (A10), we find

$$
M_{n m}(\gamma)=\frac{4}{\pi} \sum_{k=0}^{\infty} \frac{Q_{m k}(\gamma) Q_{n k}(\gamma)}{2 k+1}
$$

where $Q_{m k}(\gamma)$ are given by (A10) for $\gamma>1$. Correspondingly,

$$
f_{m}(\gamma)=\frac{4}{\pi} \sum_{k=0}^{\infty} \frac{Q_{0 k}(\gamma) Q_{m k}(\gamma)}{2 k+1}
$$

The integrals $Q_{n k}(\gamma)$ in (A10) are evaluated numerically for given modes $\psi_{n}(y)$ of the elastic plate. The function $y(0, \eta)$ of the conformal mapping is given by

$$
y(0, \eta)=\frac{2 \gamma}{\pi} \arcsin \left[\sin \left(\frac{\pi \eta}{2}\right) \sin \left(\frac{\pi}{2 \gamma}\right)\right]
$$

where $\gamma>1$. Integrating by parts in (A2) and (A10), we obtain that $Q_{n k}(\gamma)$ are of order $O\left(k^{-1}\right)$ for $\gamma<1$ and of order $O\left(k^{-4}\right)$ for $\gamma>1$, where $n$ is finite and $k \rightarrow \infty$. Therefore, the terms in the series (A3) and (A11) decay as $O\left(k^{-3}\right)$ for (A3) and $O\left(k^{-9}\right)$ for (A11) when $k \rightarrow \infty$. 


\section{Appendix B: Orthogonality of the normal modes}

Normal modes of an elastic plate of variable thickness $h(y)$ are solutions of the spectral problem (22), (23) in non-dimensional variables. The boundary conditions (23) imply that the lower end of the plate, $y=0$, is clamped, and the upper end of the plate, $y=1$, is free of stresses. In order to derive the orthogonality condition (24), we multiply both sides of equation (22), where $n \geq 1$, by $\psi_{m}(y)$, where $m \geq 1$, and integrate in $y$ along the plate, $0<y<1$. Integrating by part we find

$$
\left.\psi_{m}(y) \frac{\mathrm{d}}{\mathrm{d} y}\left(h^{3}(y) \frac{\mathrm{d}^{2} \psi_{n}}{\mathrm{~d} y^{2}}\right)\right|_{0} ^{1}-\int_{0}^{1} \frac{\mathrm{d}}{\mathrm{d} y}\left(h^{3}(y) \frac{\mathrm{d}^{2} \psi_{n}}{\mathrm{~d} y^{2}}\right) \frac{\mathrm{d} \psi_{m}}{\mathrm{~d} y} \mathrm{~d} y=\lambda_{n}^{4} \int_{0}^{1} h(y) \psi_{n}(y) \psi_{m}(y) \mathrm{d} y
$$

where the first term on the left-hand side of (B1) is equal to zero due to the boundary conditions (23). Then we integrate the left-hand side integral in (B1) by parts again,

$$
-\left.\left(h^{3}(y) \frac{\mathrm{d}^{2} \psi_{n}}{\mathrm{~d} y^{2}}\right) \frac{\mathrm{d} \psi_{m}}{\mathrm{~d} y}\right|_{0} ^{1}+\int_{0}^{1} h^{3}(y) \frac{\mathrm{d}^{2} \psi_{n}}{\mathrm{~d} y^{2}} \frac{\mathrm{d}^{2} \psi_{m}}{\mathrm{~d} y^{2}} \mathrm{~d} y=\lambda_{n}^{4} \int_{0}^{1} h(y) \psi_{n}(y) \psi_{m}(y) \mathrm{d} y .
$$

Here the first term on the left-hand side of (B2) is equal to zero due to the boundary conditions (23) and then

$$
\int_{0}^{1} h^{3}(y) \frac{\mathrm{d}^{2} \psi_{n}}{\mathrm{~d} y^{2}} \frac{\mathrm{d}^{2} \psi_{m}}{\mathrm{~d} y^{2}} \mathrm{~d} y=\lambda_{n}^{4} \int_{0}^{1} h(y) \psi_{n}(y) \psi_{m}(y) \mathrm{d} y .
$$

Changing $n$ to $m$ and $m$ to $n$ in (B3), we derive another equation,

$$
\int_{0}^{1} h^{3}(y) \frac{\mathrm{d}^{2} \psi_{m}}{\mathrm{~d} y^{2}} \frac{\mathrm{d}^{2} \psi_{n}}{\mathrm{~d} y^{2}} \mathrm{~d} y=\lambda_{m}^{4} \int_{0}^{1} h(y) \psi_{m}(y) \psi_{n}(y) \mathrm{d} y
$$

where $\lambda_{m} \neq \lambda_{n}$ for $n \neq m$. Subtracting (B4) from (B3), we obtain

$$
\left(\lambda_{n}^{4}-\lambda_{m}^{4}\right) \int_{0}^{1} h(y) \psi_{n}(y) \psi_{m}(y) \mathrm{d} y=0
$$

which provides the orthogonality condition (24) where $n \neq m$. Here we assume that different modes $\psi_{n}(y)$ correspond to different values of the spectral parameter $\lambda_{n}$. The modes $\psi_{n}(y)$ are defined as solutions of the boundary problem (22), (23) up to a constant factor. It is convenient to normalise the modes choosing this constant in such a way that the integrals in (B5) are equal to one where $n=m$. 